\title{
Palladium-Catalyzed syn-Stereocontrolled Ring-Opening of Oxabicyclic Alkenes with Sodium Arylsulfinates
}

\author{
Yue Li, Wen Yang, Guo Cheng, and Dingqiao Yang ${ }^{*}$
}

Key Laboratory of Theoretical Chemistry of Environment, Ministry of Education, School of Chemistry and Environment, South China Normal University, Guangzhou 510006, People's

Republic of China

*E-mail: yangdq@scnu.edu.cn (D.Y.). Phone: +86 20 31040403; Fax: +86 2031040403.

\section{Supporting Information}

Table of Contents

Page

1. General remarks

2. Crystal structure and data of $\mathbf{3 d a}$

3. Copies of ${ }^{1} \mathrm{H},{ }^{13} \mathrm{C}$ and ${ }^{19} \mathrm{~F}$ NMR spectra 


\section{General Remarks}

Unless otherwise indicated, all reagents were purchased from commercial suppliers and used without additional purification. Toluene was distilled from sodium benzophenone ketyl and $\mathrm{CHCl}_{3}$ was distilled from calcium hydride prior to use. Chromatographic grade $i-\mathrm{PrOH}$, 1,2-dichloroethane (DCE) and super dry solvent $\mathrm{CH}_{3} \mathrm{CN}, \mathrm{N}, \mathrm{N}$-dimethylformamide (DMF) were used without any pretreatment. Substrates 1a-e were readily prepared by the Diels-Alder reactions according to literature procedures. ${ }^{1}$ Sodium sulfinates $\mathbf{2 a}, \mathbf{2 b}$ and $\mathbf{2 f}$ were purchased from commercial suppliers and the remaining sodium arylsulfinates were prepared according to known literature procedures. ${ }^{2}{ }^{1} \mathrm{H}$ and ${ }^{13} \mathrm{C}\left\{{ }^{1} \mathrm{H}\right\}$ NMR spectra were recorded at $400 / 500 / 600 \mathrm{MHz}$ and $100 / 125 \mathrm{MHz}$ at $25{ }^{\circ} \mathrm{C}$ in $\mathrm{CDCl}_{3}$, respectively. Spectral data are reported as follows: chemical shift $(\delta, \mathrm{ppm})$; multiplicity (s-singlet, d-doublet, t-triplet, q-quadruplet, m-multiplet); coupling constants $(J, \mathrm{~Hz})$ and number of protons. HRMS (ion trap) were obtained from mass spectrometer (ESI) and MS were recorded using EI at $70 \mathrm{eV}$. Melting points were uncorrected. The crystal structure determination was carried out by X-ray diffraction apparatus.

\section{REFERENCES}

(1) (a) Best, W. M.; Collins, P. A.; McCulloch, R. K.; Wege, D. Aust. J. Chem. 1982, 35, 843-848. (b) Hart, H.; Lai, C.-Y.; Nwokogu, G. C.; Shamouilian, S. Tetrahedron 1987, 43, 5203-5224. (c) Lautens, M.; Fagnou, K.; Yang, D.-Q. J. Am. Chem. Soc. 2003, 125, 14884-14892. (d) Villeneuve, K.; Tam, W. Organometallics 2007, 26, 6082-6090.

(2) (a) Pandya, V. G.; Mhaske, S. B. Org. Lett. 2014, 16, 3836-3839. (b) Umierski N.; Manolikakes, G. Org. Lett. 2013, 15, 188-191. (c) Zhou, X.-Y.; Luo, J.-Y.; Liu, J.; Peng, S.-M.; Deng, G.-J. Org. Lett. 2011, 13, 1432-1435. 
2. Crystal structure and data of 3 da with thermal ellipsoid at $50 \%$ probability level (CCDC 1441948)

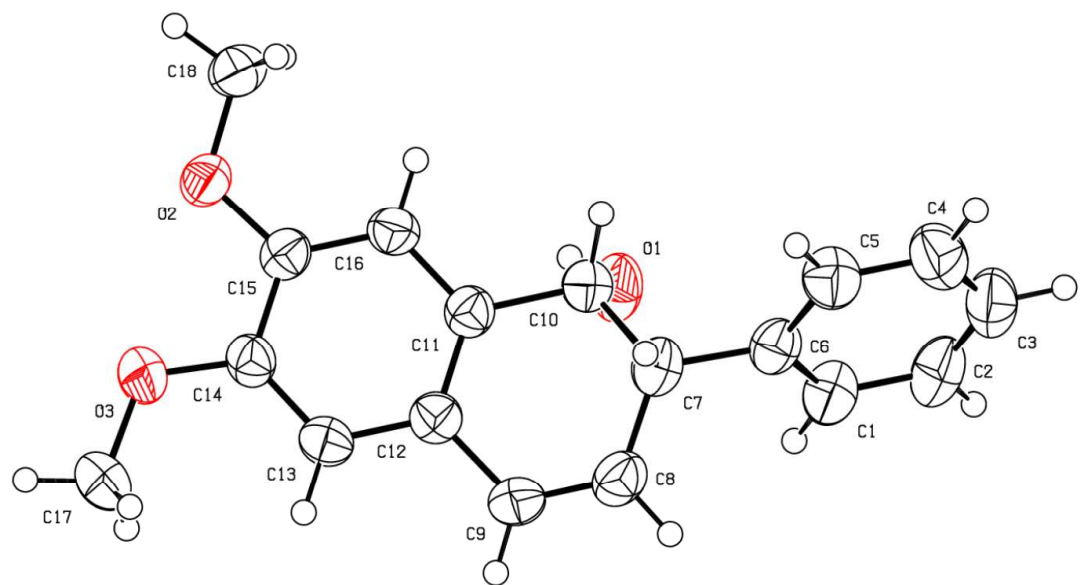

Table 1. Crystal data and structure refinement for $3 d a$.

Identification code

Empirical formula

Formula weight

Temperature

Wavelength

Crystal system, space group

Unit cell dimensions

Volume

Z, Calculated density

Absorption coefficient

$\mathrm{F}(000)$

Crystal size

Theta range for data collection 3da

$\mathrm{C}_{18} \mathrm{H}_{18} \mathrm{O}_{3}$

282.32

298(2) K

$0.71073 \mathrm{~A}$

Triclinic, P-1

$\mathrm{a}=7.641(9) \mathrm{A} \quad$ alpha $=87.78(2) \mathrm{deg}$.

$\mathrm{b}=9.967(12) \mathrm{A} \quad$ beta $=80.78(2) \mathrm{deg}$.

$\mathrm{c}=10.058(13) \mathrm{A} \quad$ gamma $=67.547(18) \mathrm{deg}$.

$698.6(15) \mathrm{A}^{\wedge} 3$

2, $\quad 1.342 \mathrm{Mg} / \mathrm{m}^{\wedge} 3$

$0.090 \mathrm{~mm}^{\wedge}-1$

300

$0.35 \times 0.32 \times 0.30 \mathrm{~mm}$

2.05 to $25.10 \mathrm{deg}$. 
Limiting indices

Reflections collected / unique

Completeness to theta $=25.10$

Max. and min. transmission

Refinement method

Data / restraints / parameters

Goodness-of-fit on $\mathrm{F}^{\wedge} 2$

Final $\mathrm{R}$ indices $[\mathrm{I}>2 \operatorname{sigma}(\mathrm{I})]$

$\mathrm{R}$ indices (all data)

Largest diff. peak and hole $-9<=\mathrm{h}<=6,-10<=\mathrm{k}<=11,-11<=\mathrm{l}<=8$

$3476 / 2423[\mathrm{R}($ int $)=0.0230]$

$97.8 \%$

0.9734 and 0.9690

Full-matrix least-squares on $\mathrm{F}^{\wedge} 2$

2423 / 0 / 193

1.025

$\mathrm{R} 1=0.0452, \mathrm{wR} 2=0.1151$

$\mathrm{R} 1=0.0566, \mathrm{wR} 2=0.1273$

0.191 and -0.307 e. $\mathrm{A}^{\wedge}-3$ 
Table 2. Atomic coordinates ( $\left.x \mathbf{1 0}^{\wedge} 4\right)$ and equivalent isotropic displacement parameters $\left(A^{\wedge} 2 \times 10^{\wedge} 3\right)$ for $3 \mathrm{da} . \mathrm{U}(\mathrm{eq})$ is defined as one third of the trace of the orthogonalized $\mathrm{U}^{\mathrm{ij}}$ tensor.

\begin{tabular}{|c|c|c|c|c|}
\hline & $\mathrm{x}$ & $\mathrm{y}$ & $\mathrm{z}$ & $\mathrm{U}(\mathrm{eq})$ \\
\hline $\mathrm{O}(2)$ & $447(2)$ & 11501(1) & $6390(1)$ & $47(1)$ \\
\hline$C(12)$ & $4609(2)$ & $7602(2)$ & $4961(2)$ & $42(1)$ \\
\hline$C(16)$ & $2556(2)$ & $9987(2)$ & $4518(2)$ & $43(1)$ \\
\hline$C(15)$ & $1827(2)$ & $10243(2)$ & $5856(2)$ & $41(1)$ \\
\hline $\mathrm{O}(3)$ & $1676(2)$ & $9518(1)$ & $8078(1)$ & $56(1)$ \\
\hline $\mathrm{O}(1)$ & $3335(2)$ & $7776(2)$ & $2065(1)$ & $58(1)$ \\
\hline$C(13)$ & $3885(2)$ & $7867(2)$ & $6321(2)$ & $44(1)$ \\
\hline$C(14)$ & $2497(2)$ & $9170(2)$ & $6777(2)$ & $42(1)$ \\
\hline $\mathrm{C}(11)$ & $3934(2)$ & $8675(2)$ & $4053(2)$ & $42(1)$ \\
\hline$C(6)$ & $7148(2)$ & $6845(2)$ & $786(2)$ & $45(1)$ \\
\hline$C(10)$ & $4548(2)$ & $8352(2)$ & $2570(2)$ & $46(1)$ \\
\hline $\mathrm{C}(9)$ & $6026(2)$ & $6219(2)$ & $4452(2)$ & $48(1)$ \\
\hline $\mathrm{C}(8)$ & $6899(2)$ & $6036(2)$ & $3196(2)$ & $51(1)$ \\
\hline$C(7)$ & $6578(2)$ & $7261(2)$ & $2259(2)$ & $47(1)$ \\
\hline $\mathrm{C}(1)$ & $6888(3)$ & $5708(2)$ & $255(2)$ & $56(1)$ \\
\hline $\mathrm{C}(18)$ & $-165(3)$ & $12654(2)$ & $5500(2)$ & $55(1)$ \\
\hline$C(2)$ & $7384(3)$ & $5370(2)$ & $-1099(2)$ & $64(1)$ \\
\hline$C(17)$ & $2460(3)$ & $8530(2)$ & $9070(2)$ & $67(1)$ \\
\hline$C(5)$ & $7938(3)$ & $7629(2)$ & $-80(2)$ & $57(1)$ \\
\hline$C(4)$ & $8432(3)$ & $7290(2)$ & $-1430(2)$ & $65(1)$ \\
\hline $\mathrm{C}(3)$ & $8145(3)$ & $6166(2)$ & $-1941(2)$ & $64(1)$ \\
\hline
\end{tabular}


Table 3. Bond lengths $[\mathrm{A}]$ and angles [deg] for $3 \mathrm{da}$.

\begin{tabular}{|c|c|}
\hline $\mathrm{O}(2)-\mathrm{C}(15)$ & $1.354(2)$ \\
\hline $\mathrm{O}(2)-\mathrm{C}(18)$ & $1.409(2)$ \\
\hline$C(12)-C(11)$ & $1.377(3)$ \\
\hline $\mathrm{C}(12)-\mathrm{C}(13)$ & $1.385(3)$ \\
\hline $\mathrm{C}(12)-\mathrm{C}(9)$ & $1.440(3)$ \\
\hline$C(16)-C(15)$ & $1.365(3)$ \\
\hline$C(16)-C(11)$ & $1.370(3)$ \\
\hline $\mathrm{C}(16)-\mathrm{H}(16)$ & 0.9300 \\
\hline$C(15)-C(14)$ & $1.386(3)$ \\
\hline $\mathrm{O}(3)-\mathrm{C}(14)$ & $1.354(2)$ \\
\hline $\mathrm{O}(3)-\mathrm{C}(17)$ & $1.410(2)$ \\
\hline $\mathrm{O}(1)-\mathrm{C}(10)$ & $1.424(2)$ \\
\hline $\mathrm{O}(1)-\mathrm{H}(1)$ & 0.8200 \\
\hline$C(13)-C(14)$ & $1.364(3)$ \\
\hline $\mathrm{C}(13)-\mathrm{H}(13)$ & 0.9300 \\
\hline $\mathrm{C}(11)-\mathrm{C}(10)$ & $1.499(3)$ \\
\hline$C(6)-C(1)$ & $1.364(3)$ \\
\hline$C(6)-C(5)$ & $1.369(3)$ \\
\hline$C(6)-C(7)$ & $1.503(3)$ \\
\hline$C(10)-C(7)$ & $1.506(3)$ \\
\hline $\mathrm{C}(10)-\mathrm{H}(10)$ & 0.9800 \\
\hline $\mathrm{C}(9)-\mathrm{C}(8)$ & $1.315(3)$ \\
\hline $\mathrm{C}(9)-\mathrm{H}(9)$ & 0.9300 \\
\hline$C(8)-C(7)$ & $1.481(3)$ \\
\hline $\mathrm{C}(8)-\mathrm{H}(8)$ & 0.9300 \\
\hline $\mathrm{C}(7)-\mathrm{H}(7)$ & 0.9800 \\
\hline$C(1)-C(2)$ & $1.374(3)$ \\
\hline $\mathrm{C}(1)-\mathrm{H}(1 \mathrm{~A})$ & 0.9300 \\
\hline & \\
\hline
\end{tabular}




\begin{tabular}{|c|c|}
\hline $\mathrm{C}(18)-\mathrm{H}(18 \mathrm{~A})$ & 0.9600 \\
\hline $\mathrm{C}(18)-\mathrm{H}(18 \mathrm{~B})$ & 0.9600 \\
\hline $\mathrm{C}(18)-\mathrm{H}(18 \mathrm{C})$ & 0.9600 \\
\hline $\mathrm{C}(2)-\mathrm{C}(3)$ & $1.352(3)$ \\
\hline $\mathrm{C}(2)-\mathrm{H}(2)$ & 0.9300 \\
\hline $\mathrm{C}(17)-\mathrm{H}(17 \mathrm{~A})$ & 0.9600 \\
\hline $\mathrm{C}(17)-\mathrm{H}(17 \mathrm{~B})$ & 0.9600 \\
\hline $\mathrm{C}(17)-\mathrm{H}(17 \mathrm{C})$ & 0.9600 \\
\hline $\mathrm{C}(5)-\mathrm{C}(4)$ & $1.370(3)$ \\
\hline $\mathrm{C}(5)-\mathrm{H}(5)$ & 0.9300 \\
\hline $\mathrm{C}(4)-\mathrm{C}(3)$ & $1.354(3)$ \\
\hline $\mathrm{C}(4)-\mathrm{H}(4)$ & 0.9300 \\
\hline $\mathrm{C}(3)-\mathrm{H}(3)$ & 0.9300 \\
\hline $\mathrm{C}(15)-\mathrm{O}(2)-\mathrm{C}(18)$ & $116.74(15)$ \\
\hline$C(11)-C(12)-C(13)$ & $119.66(16)$ \\
\hline $\mathrm{C}(11)-\mathrm{C}(12)-\mathrm{C}(9)$ & $118.27(17)$ \\
\hline $\mathrm{C}(13)-\mathrm{C}(12)-\mathrm{C}(9)$ & $122.05(15)$ \\
\hline$C(15)-C(16)-C(11)$ & $121.37(15)$ \\
\hline $\mathrm{C}(15)-\mathrm{C}(16)-\mathrm{H}(16)$ & 119.3 \\
\hline $\mathrm{C}(11)-\mathrm{C}(16)-\mathrm{H}(16)$ & 119.3 \\
\hline $\mathrm{O}(2)-\mathrm{C}(15)-\mathrm{C}(16)$ & $124.85(15)$ \\
\hline $\mathrm{O}(2)-\mathrm{C}(15)-\mathrm{C}(14)$ & $115.13(16)$ \\
\hline$C(16)-C(15)-C(14)$ & $120.02(16)$ \\
\hline $\mathrm{C}(14)-\mathrm{O}(3)-\mathrm{C}(17)$ & $117.86(16)$ \\
\hline $\mathrm{C}(10)-\mathrm{O}(1)-\mathrm{H}(1)$ & 109.5 \\
\hline $\mathrm{C}(14)-\mathrm{C}(13)-\mathrm{C}(12)$ & 121.11(15) \\
\hline $\mathrm{C}(14)-\mathrm{C}(13)-\mathrm{H}(13)$ & 119.4 \\
\hline $\mathrm{C}(12)-\mathrm{C}(13)-\mathrm{H}(13)$ & 119.4 \\
\hline $\mathrm{O}(3)-\mathrm{C}(14)-\mathrm{C}(13)$ & $125.35(15)$ \\
\hline $\mathrm{O}(3)-\mathrm{C}(14)-\mathrm{C}(15)$ & $115.82(16)$ \\
\hline
\end{tabular}




\begin{tabular}{|c|c|}
\hline$C(13)-C(14)-C(15)$ & $118.83(17)$ \\
\hline$C(16)-C(11)-C(12)$ & 118.99(17) \\
\hline$C(16)-C(11)-C(10)$ & $120.57(15)$ \\
\hline$C(12)-C(11)-C(10)$ & $120.12(16)$ \\
\hline$C(1)-C(6)-C(5)$ & $117.58(19)$ \\
\hline $\mathrm{C}(1)-\mathrm{C}(6)-\mathrm{C}(7)$ & $122.46(16)$ \\
\hline$C(5)-C(6)-C(7)$ & 119.96(17) \\
\hline $\mathrm{O}(1)-\mathrm{C}(10)-\mathrm{C}(11)$ & $110.30(14)$ \\
\hline $\mathrm{O}(1)-\mathrm{C}(10)-\mathrm{C}(7)$ & $107.93(16)$ \\
\hline $\mathrm{C}(11)-\mathrm{C}(10)-\mathrm{C}(7)$ & $111.61(14)$ \\
\hline $\mathrm{O}(1)-\mathrm{C}(10)-\mathrm{H}(10)$ & 109.0 \\
\hline $\mathrm{C}(11)-\mathrm{C}(10)-\mathrm{H}(10)$ & 109.0 \\
\hline $\mathrm{C}(7)-\mathrm{C}(10)-\mathrm{H}(10)$ & 109.0 \\
\hline $\mathrm{C}(8)-\mathrm{C}(9)-\mathrm{C}(12)$ & $121.57(16)$ \\
\hline $\mathrm{C}(8)-\mathrm{C}(9)-\mathrm{H}(9)$ & 119.2 \\
\hline $\mathrm{C}(12)-\mathrm{C}(9)-\mathrm{H}(9)$ & 119.2 \\
\hline$C(9)-C(8)-C(7)$ & $121.85(16)$ \\
\hline $\mathrm{C}(9)-\mathrm{C}(8)-\mathrm{H}(8)$ & 119.1 \\
\hline $\mathrm{C}(7)-\mathrm{C}(8)-\mathrm{H}(8)$ & 119.1 \\
\hline$C(8)-C(7)-C(6)$ & $115.59(16)$ \\
\hline$C(8)-C(7)-C(10)$ & $110.64(15)$ \\
\hline$C(6)-C(7)-C(10)$ & $111.79(14)$ \\
\hline $\mathrm{C}(8)-\mathrm{C}(7)-\mathrm{H}(7)$ & 106.0 \\
\hline $\mathrm{C}(6)-\mathrm{C}(7)-\mathrm{H}(7)$ & 106.0 \\
\hline $\mathrm{C}(10)-\mathrm{C}(7)-\mathrm{H}(7)$ & 106.0 \\
\hline $\mathrm{C}(6)-\mathrm{C}(1)-\mathrm{C}(2)$ & $121.29(19)$ \\
\hline $\mathrm{C}(6)-\mathrm{C}(1)-\mathrm{H}(1 \mathrm{~A})$ & 119.4 \\
\hline $\mathrm{C}(2)-\mathrm{C}(1)-\mathrm{H}(1 \mathrm{~A})$ & 119.4 \\
\hline $\mathrm{O}(2)-\mathrm{C}(18)-\mathrm{H}(18 \mathrm{~A})$ & 109.5 \\
\hline \multirow[t]{2}{*}{$\mathrm{O}(2)-\mathrm{C}(18)-\mathrm{H}(18 \mathrm{~B})$} & 109.5 \\
\hline & S8 \\
\hline
\end{tabular}




\begin{tabular}{|c|c|}
\hline H(18A)-C(18)-H(18B) & 109.5 \\
\hline $\mathrm{O}(2)-\mathrm{C}(18)-\mathrm{H}(18 \mathrm{C})$ & 109.5 \\
\hline $\mathrm{H}(18 \mathrm{~A})-\mathrm{C}(18)-\mathrm{H}(18 \mathrm{C})$ & 109.5 \\
\hline $\mathrm{H}(18 \mathrm{~B})-\mathrm{C}(18)-\mathrm{H}(18 \mathrm{C})$ & 109.5 \\
\hline $\mathrm{C}(3)-\mathrm{C}(2)-\mathrm{C}(1)$ & $120.3(2)$ \\
\hline $\mathrm{C}(3)-\mathrm{C}(2)-\mathrm{H}(2)$ & 119.9 \\
\hline $\mathrm{C}(1)-\mathrm{C}(2)-\mathrm{H}(2)$ & 119.9 \\
\hline $\mathrm{O}(3)-\mathrm{C}(17)-\mathrm{H}(17 \mathrm{~A})$ & 109.5 \\
\hline $\mathrm{O}(3)-\mathrm{C}(17)-\mathrm{H}(17 \mathrm{~B})$ & 109.5 \\
\hline H(17A)-C(17)-H(17B) & 109.5 \\
\hline $\mathrm{O}(3)-\mathrm{C}(17)-\mathrm{H}(17 \mathrm{C})$ & 109.5 \\
\hline $\mathrm{H}(17 \mathrm{~A})-\mathrm{C}(17)-\mathrm{H}(17 \mathrm{C})$ & 109.5 \\
\hline H(17B)-C(17)-H(17C) & 109.5 \\
\hline$C(6)-C(5)-C(4)$ & $121.0(2)$ \\
\hline $\mathrm{C}(6)-\mathrm{C}(5)-\mathrm{H}(5)$ & 119.5 \\
\hline $\mathrm{C}(4)-\mathrm{C}(5)-\mathrm{H}(5)$ & 119.5 \\
\hline$C(3)-C(4)-C(5)$ & $120.56(19)$ \\
\hline $\mathrm{C}(3)-\mathrm{C}(4)-\mathrm{H}(4)$ & 119.7 \\
\hline $\mathrm{C}(5)-\mathrm{C}(4)-\mathrm{H}(4)$ & 119.7 \\
\hline$C(2)-C(3)-C(4)$ & $119.3(2)$ \\
\hline $\mathrm{C}(2)-\mathrm{C}(3)-\mathrm{H}(3)$ & 120.4 \\
\hline $\mathrm{C}(4)-\mathrm{C}(3)-\mathrm{H}(3)$ & 120.4 \\
\hline
\end{tabular}

Symmetry transformations used to generate equivalent atoms: 
Table 4. Anisotropic displacement parameters $\left(\mathrm{A}^{\wedge} 2 \times 10^{\wedge} 3\right)$ for $3 \mathrm{da}$. The anisotropic displacement factor exponent takes the form: $-2 p^{\wedge}{ }^{\wedge}\left[h^{\wedge} 2 a^{* \wedge} 2\right.$ U11 $\left.+\ldots+2 h k a^{*} b^{*} U 12\right]$

\begin{tabular}{lrrrrrr}
\hline & $\mathrm{U} 11$ & $\mathrm{U} 22$ & $\mathrm{U} 33$ & $\mathrm{U} 23$ & $\mathrm{U} 13$ & $\mathrm{U} 12$ \\
\hline $\mathrm{O}(2)$ & $48(1)$ & $43(1)$ & $44(1)$ & $-5(1)$ & $-9(1)$ & $-9(1)$ \\
$\mathrm{C}(12)$ & $38(1)$ & $44(1)$ & $44(1)$ & $1(1)$ & $-10(1)$ & $-15(1)$ \\
$\mathrm{C}(16)$ & $48(1)$ & $39(1)$ & $41(1)$ & $4(1)$ & $-13(1)$ & $-14(1)$ \\
$\mathrm{C}(15)$ & $40(1)$ & $40(1)$ & $43(1)$ & $-4(1)$ & $-11(1)$ & $-14(1)$ \\
$\mathrm{O}(3)$ & $63(1)$ & $58(1)$ & $36(1)$ & $0(1)$ & $-8(1)$ & $-11(1)$ \\
$\mathrm{O}(1)$ & $43(1)$ & $77(1)$ & $47(1)$ & $-14(1)$ & $-10(1)$ & $-13(1)$ \\
$\mathrm{C}(13)$ & $44(1)$ & $46(1)$ & $42(1)$ & $6(1)$ & $-14(1)$ & $-14(1)$ \\
$\mathrm{C}(14)$ & $43(1)$ & $48(1)$ & $37(1)$ & $-1(1)$ & $-10(1)$ & $-16(1)$ \\
$\mathrm{C}(11)$ & $41(1)$ & $43(1)$ & $41(1)$ & $-1(1)$ & $-8(1)$ & $-15(1)$ \\
$\mathrm{C}(6)$ & $36(1)$ & $49(1)$ & $49(1)$ & $-5(1)$ & $-4(1)$ & $-14(1)$ \\
$\mathrm{C}(10)$ & $51(1)$ & $43(1)$ & $41(1)$ & $0(1)$ & $-7(1)$ & $-14(1)$ \\
$\mathrm{C}(9)$ & $43(1)$ & $43(1)$ & $52(1)$ & $5(1)$ & $-12(1)$ & $-9(1)$ \\
$\mathrm{C}(8)$ & $41(1)$ & $49(1)$ & $54(1)$ & $-5(1)$ & $-8(1)$ & $-8(1)$ \\
$\mathrm{C}(7)$ & $42(1)$ & $51(1)$ & $48(1)$ & $-7(1)$ & $-4(1)$ & $-19(1)$ \\
$\mathrm{C}(1)$ & $54(1)$ & $55(1)$ & $58(1)$ & $-9(1)$ & $1(1)$ & $-23(1)$ \\
$\mathrm{C}(18)$ & $58(1)$ & $44(1)$ & $56(1)$ & $-1(1)$ & $-13(1)$ & $-11(1)$ \\
$\mathrm{C}(2)$ & $59(1)$ & $65(1)$ & $62(1)$ & $-22(1)$ & $-2(1)$ & $-19(1)$ \\
$\mathrm{C}(17)$ & $78(1)$ & $71(1)$ & $41(1)$ & $9(1)$ & $-12(1)$ & $-16(1)$ \\
$\mathrm{C}(5)$ & $60(1)$ & $59(1)$ & $56(1)$ & $-1(1)$ & $-5(1)$ & $-27(1)$ \\
$\mathrm{C}(4)$ & $66(1)$ & $78(1)$ & $52(1)$ & $6(1)$ & $-2(1)$ & $-29(1)$ \\
$\mathrm{C}(3)$ & $51(1)$ & $81(1)$ & $47(1)$ & $-11(1)$ & $-2(1)$ & $-13(1)$ \\
\hline & & & & & & \\
\hline & & & & & & \\
\hline
\end{tabular}


Table 5. Hydrogen coordinates $\left(\begin{array}{ll}x & \left.10^{\wedge} 4\right)\end{array}\right)$ and isotropic displacement parameters $\left(A^{\wedge} 2 \times\right.$ $\left.10^{\wedge} 3\right)$ for $3 d a$.

\begin{tabular}{|c|c|c|c|c|}
\hline & $\mathrm{x}$ & $\mathrm{y}$ & $\mathrm{z}$ & $\mathrm{U}(\mathrm{eq})$ \\
\hline $\mathrm{H}(16)$ & 2108 & 10719 & 3911 & 52 \\
\hline $\mathrm{H}(1)$ & 2217 & 8233 & 2398 & 87 \\
\hline $\mathrm{H}(13)$ & 4354 & 7145 & 6933 & 53 \\
\hline $\mathrm{H}(10)$ & 4460 & 9254 & 2107 & 55 \\
\hline $\mathrm{H}(9)$ & 6331 & 5436 & 5025 & 57 \\
\hline $\mathrm{H}(8)$ & 7743 & 5112 & 2887 & 61 \\
\hline $\mathrm{H}(7)$ & 7398 & 7758 & 2454 & 56 \\
\hline $\mathrm{H}(1 \mathrm{~A})$ & 6364 & 5151 & 822 & 67 \\
\hline $\mathrm{H}(18 \mathrm{~A})$ & 910 & 12883 & 5094 & 82 \\
\hline $\mathrm{H}(18 \mathrm{~B})$ & -1117 & 13492 & 5990 & 82 \\
\hline $\mathrm{H}(18 \mathrm{C})$ & -703 & 12375 & 4811 & 82 \\
\hline $\mathrm{H}(2)$ & 7195 & 4589 & -1440 & 77 \\
\hline $\mathrm{H}(17 \mathrm{~A})$ & 2296 & 7638 & 8940 & 100 \\
\hline $\mathrm{H}(17 \mathrm{~B})$ & 1816 & 8937 & 9947 & 100 \\
\hline $\mathrm{H}(17 \mathrm{C})$ & 3802 & 8341 & 8998 & 100 \\
\hline $\mathrm{H}(5)$ & 8143 & 8405 & 255 & 69 \\
\hline $\mathrm{H}(4)$ & 8969 & 7835 & -2002 & 78 \\
\hline $\mathrm{H}(3)$ & 8468 & 5945 & -2860 & 77 \\
\hline
\end{tabular}


3. Copies of ${ }^{1} \mathrm{H},{ }^{13} \mathrm{C}$ and ${ }^{19} \mathrm{~F}$ NMR spectra

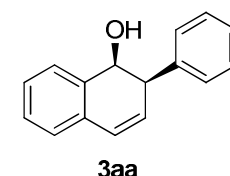

$500 \mathrm{MHz} \mathrm{CDCl}_{3}$
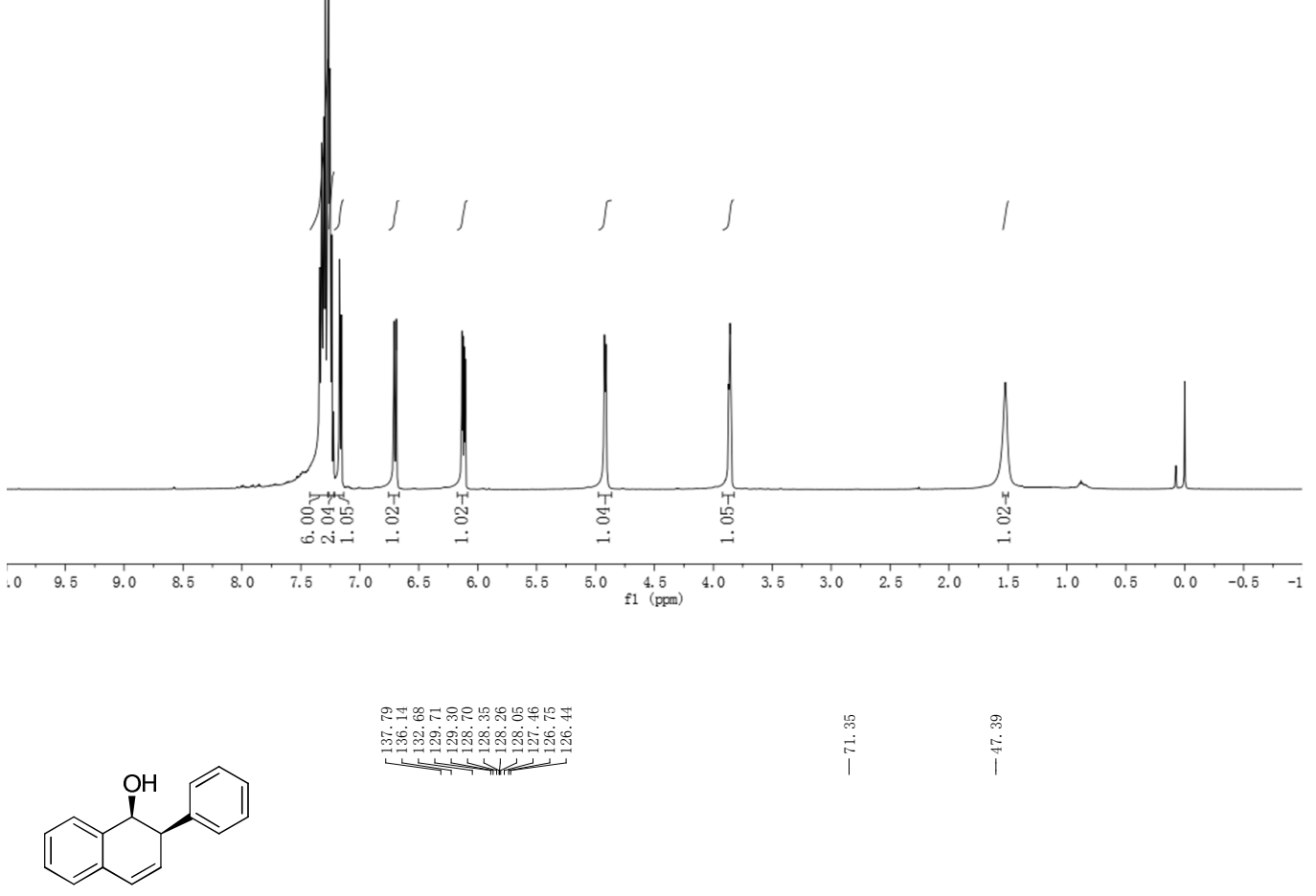

$3 a a$

$125 \mathrm{MHz}, \mathrm{CDCl}_{3}$

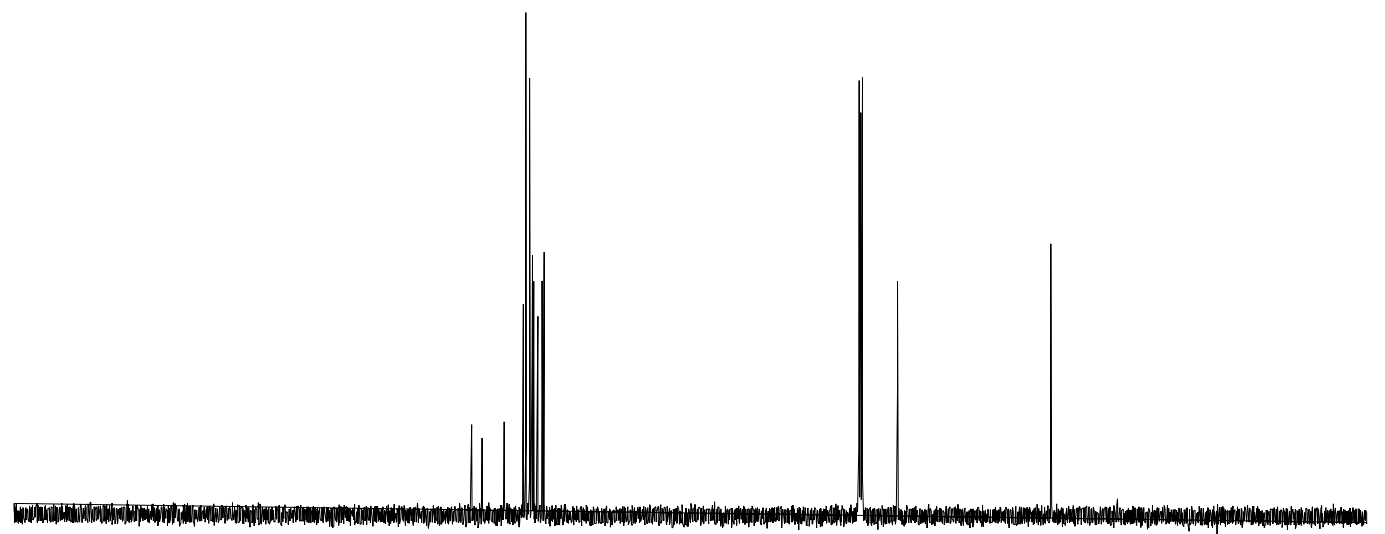



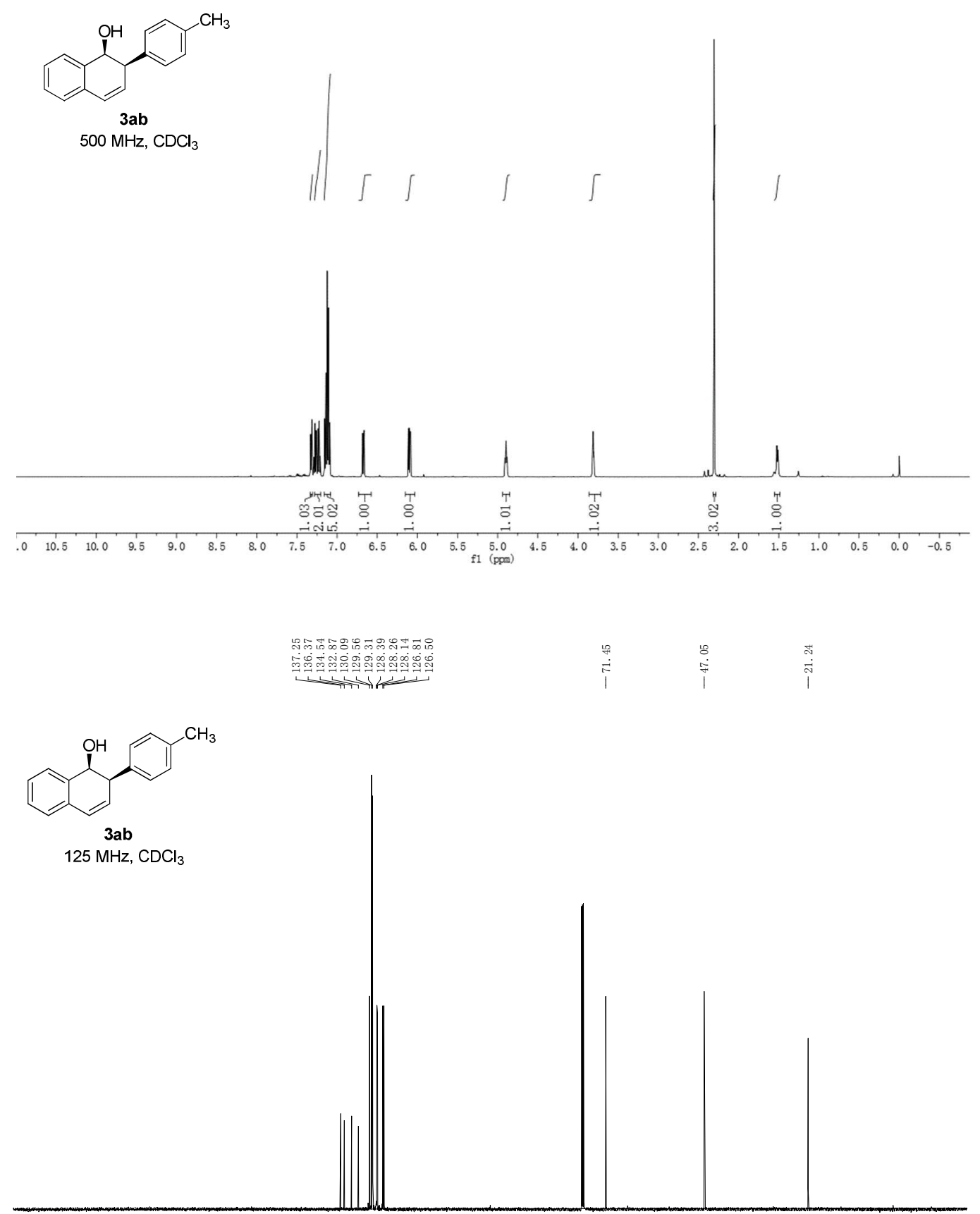

$\begin{array}{rllllllllllllllllllllllll}1 & 1 \\ 210 & 200 & 190 & 180 & 170 & 160 & 150 & 140 & 130 & 120 & 110 & 100 & 1 & 10 & 10 & 70 & 60 & 50 & 40 & 30 & 20 & 10 & 0 & -10\end{array}$ 

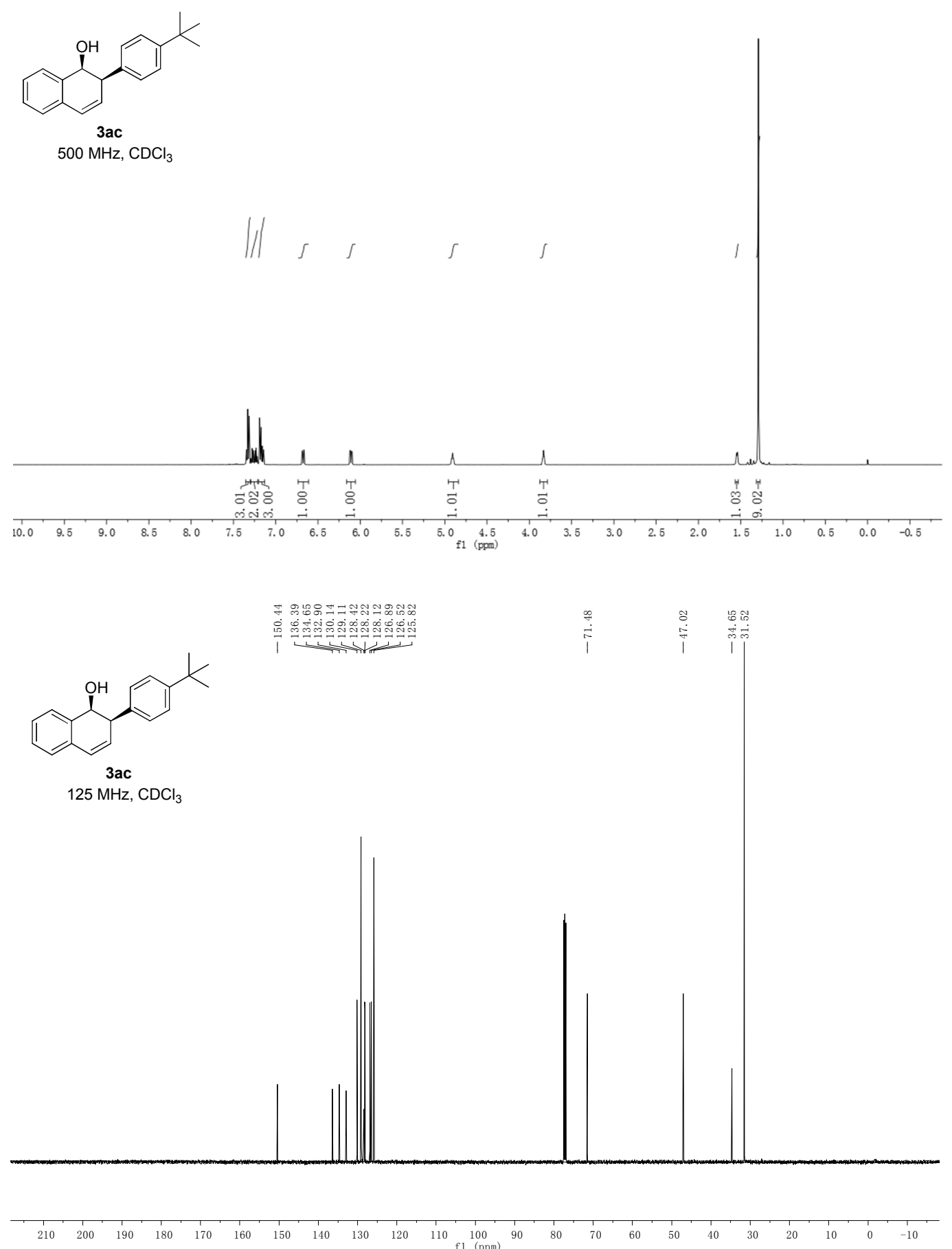

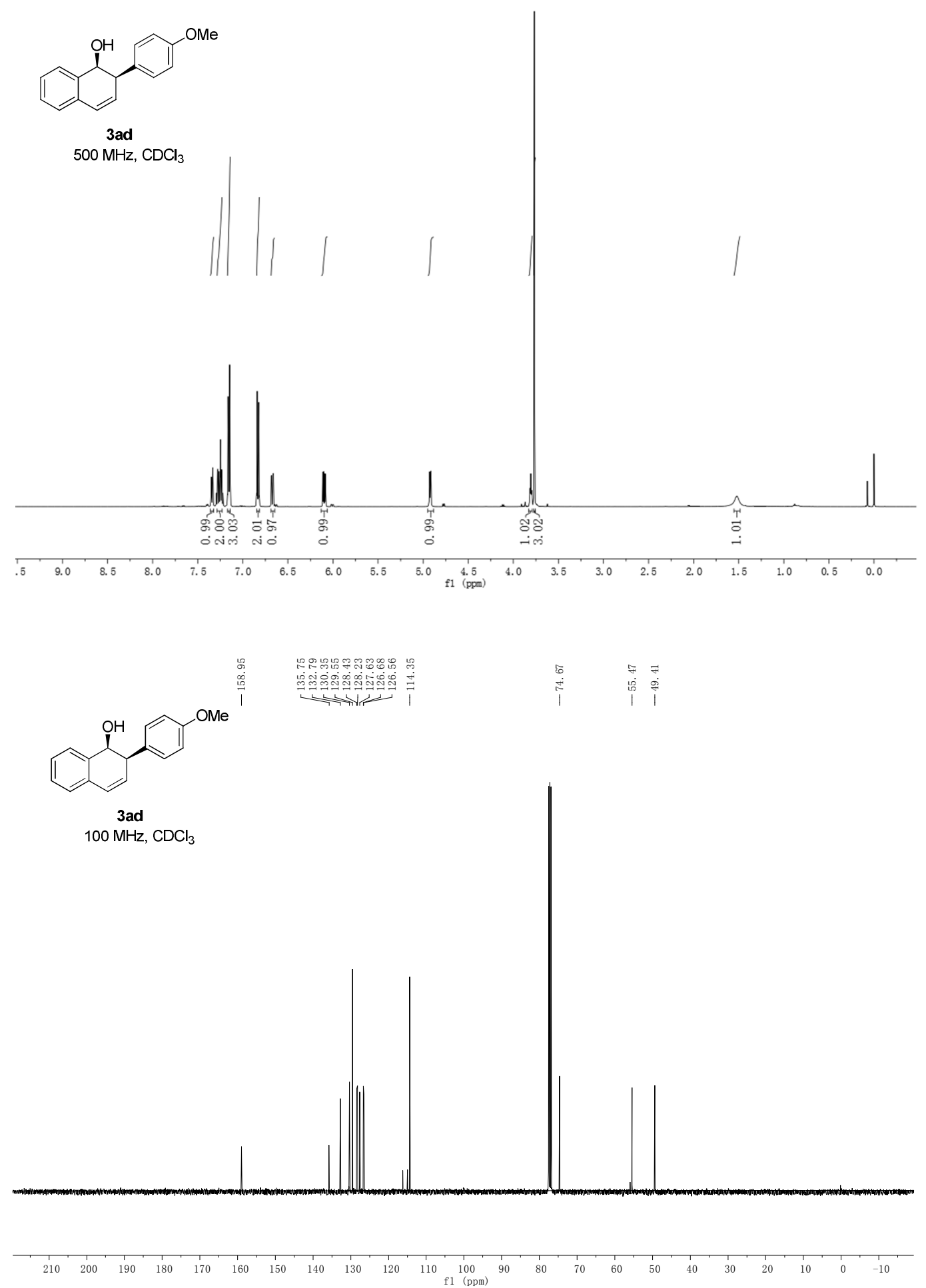

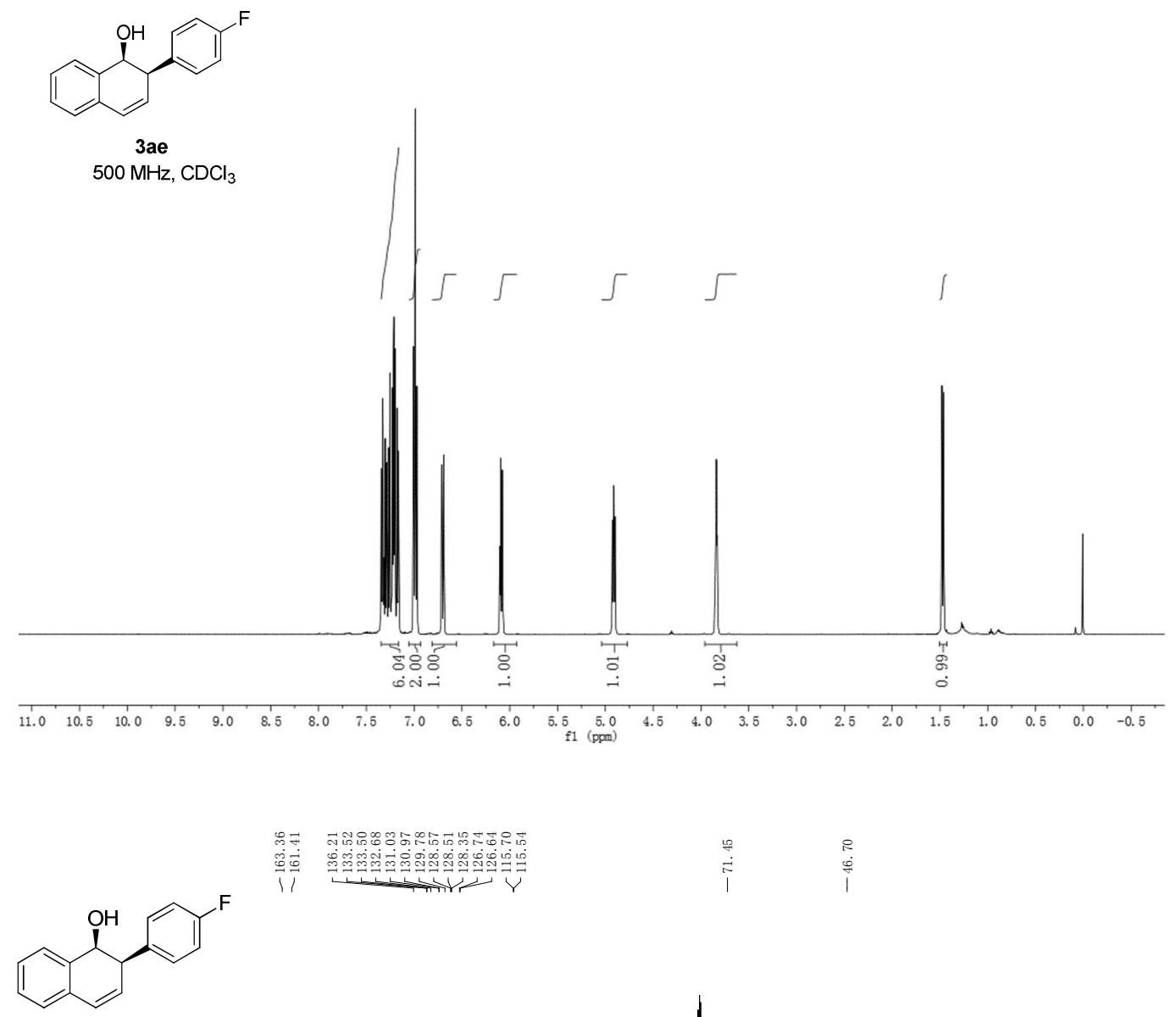

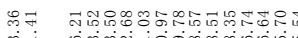

:ं

3 ae

$125 \mathrm{MHz}, \mathrm{CDCl}_{3}$
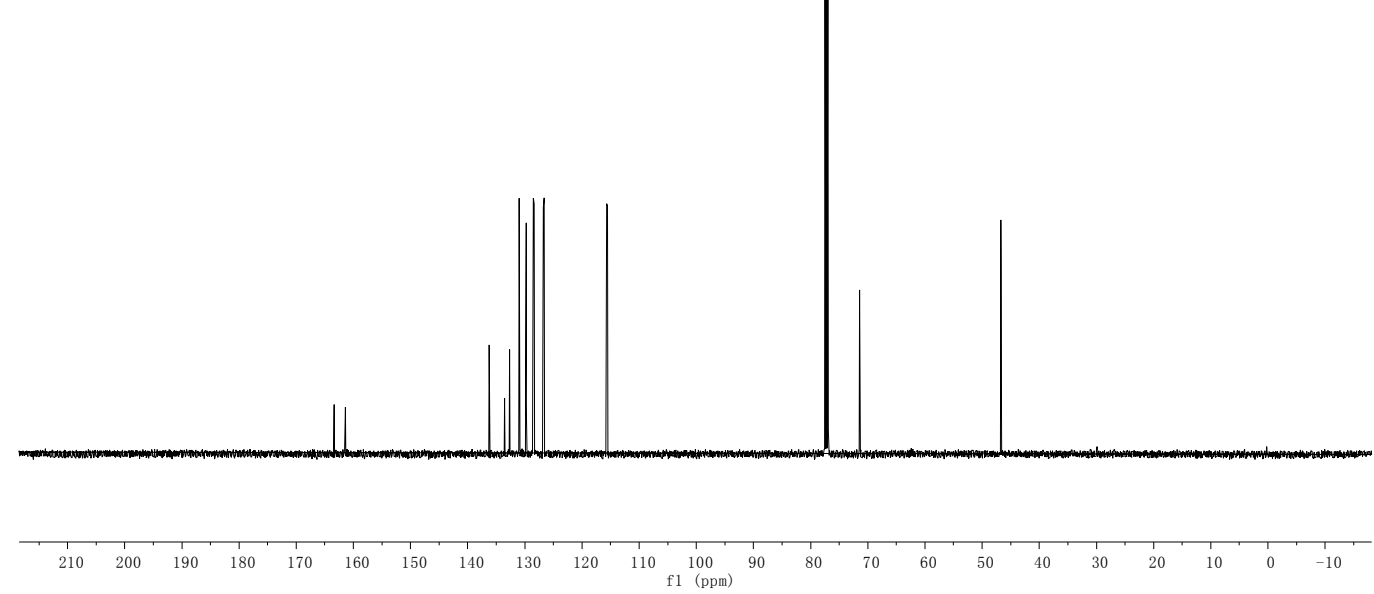


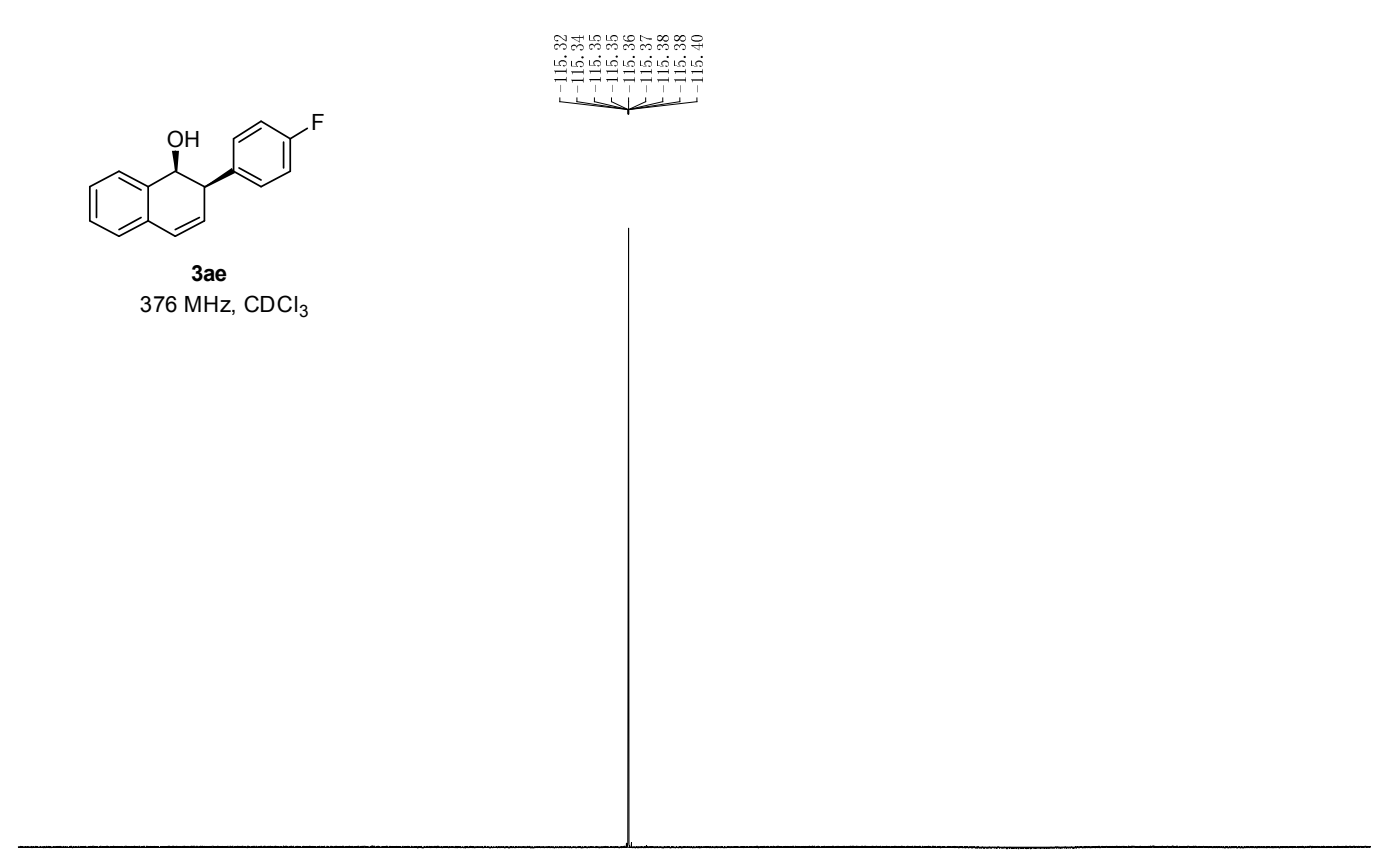

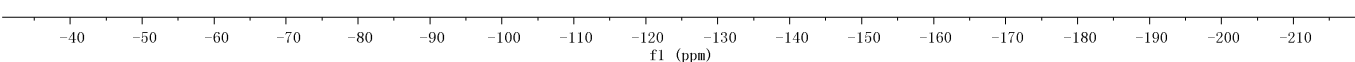



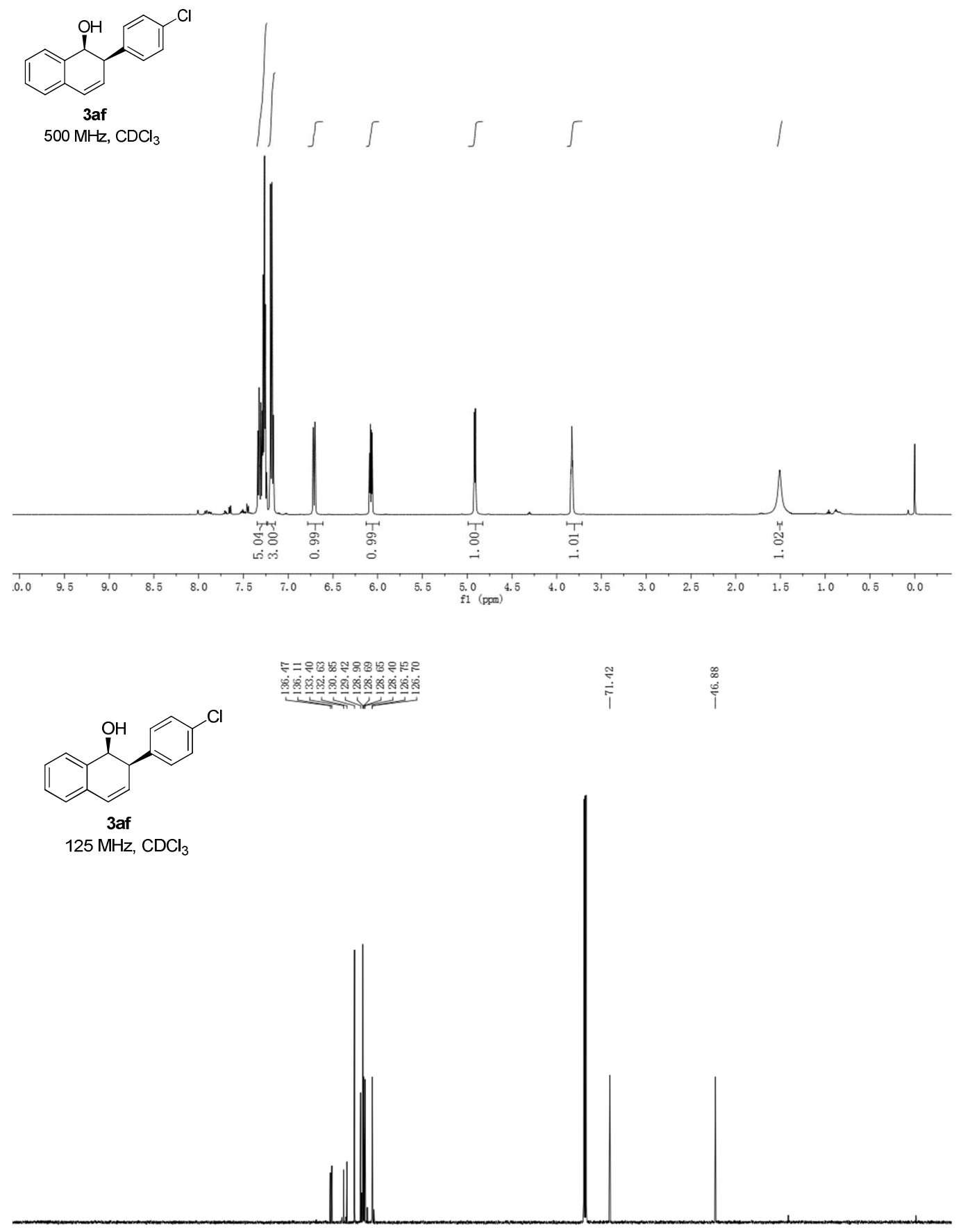

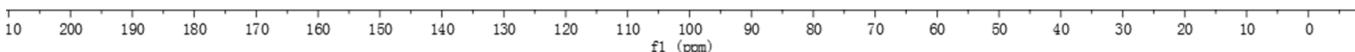



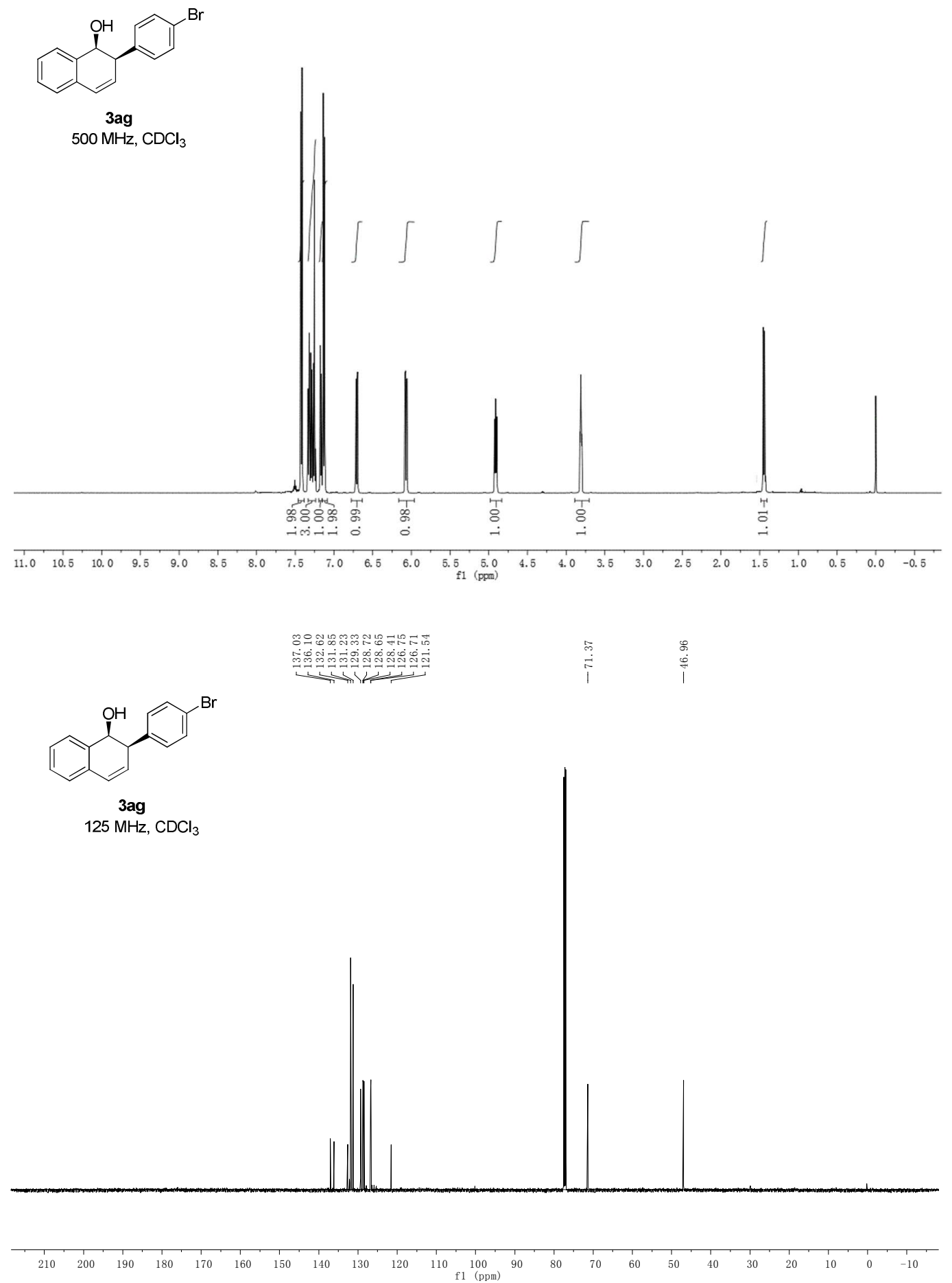

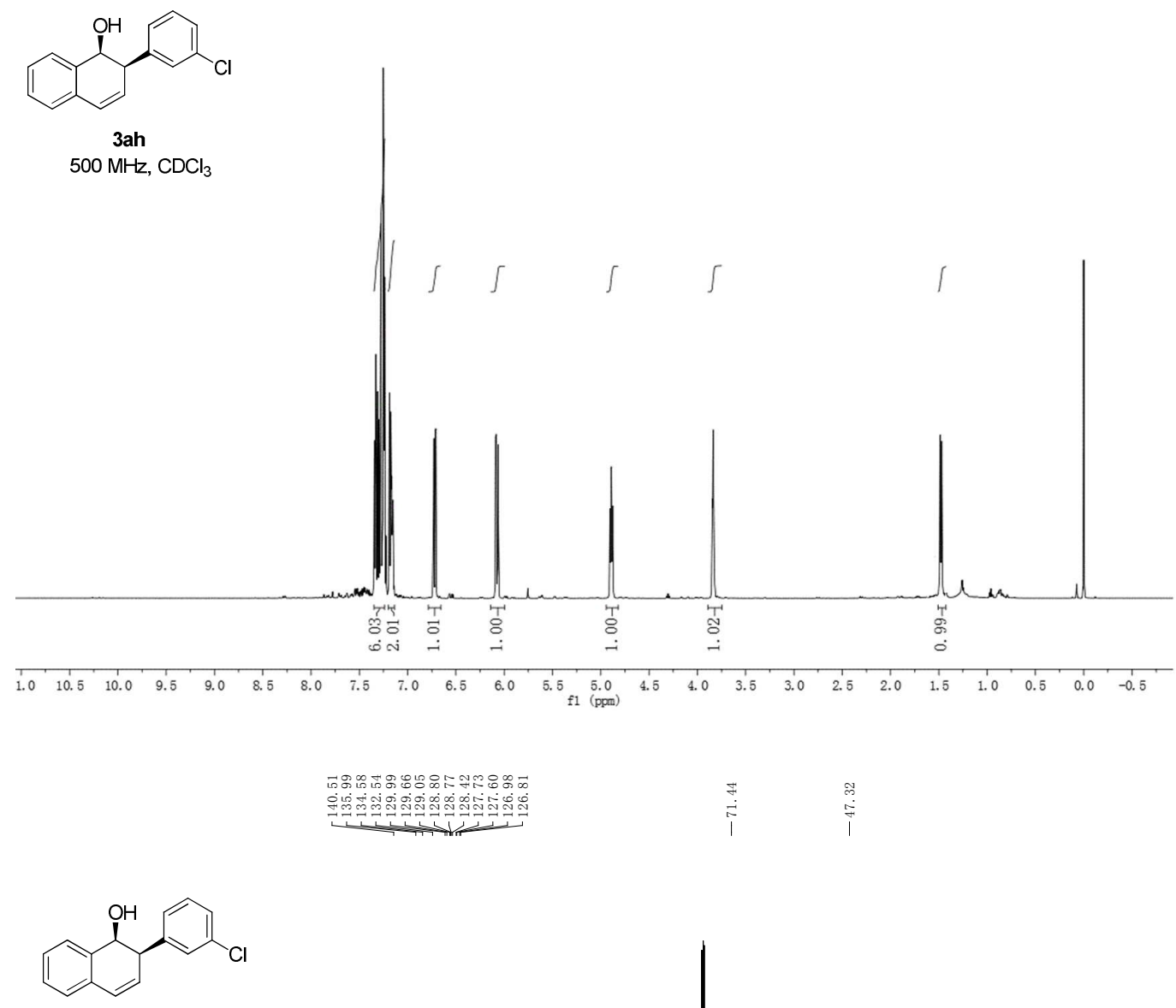

3ah

$125 \mathrm{MHz}, \mathrm{CDCl}_{3}$
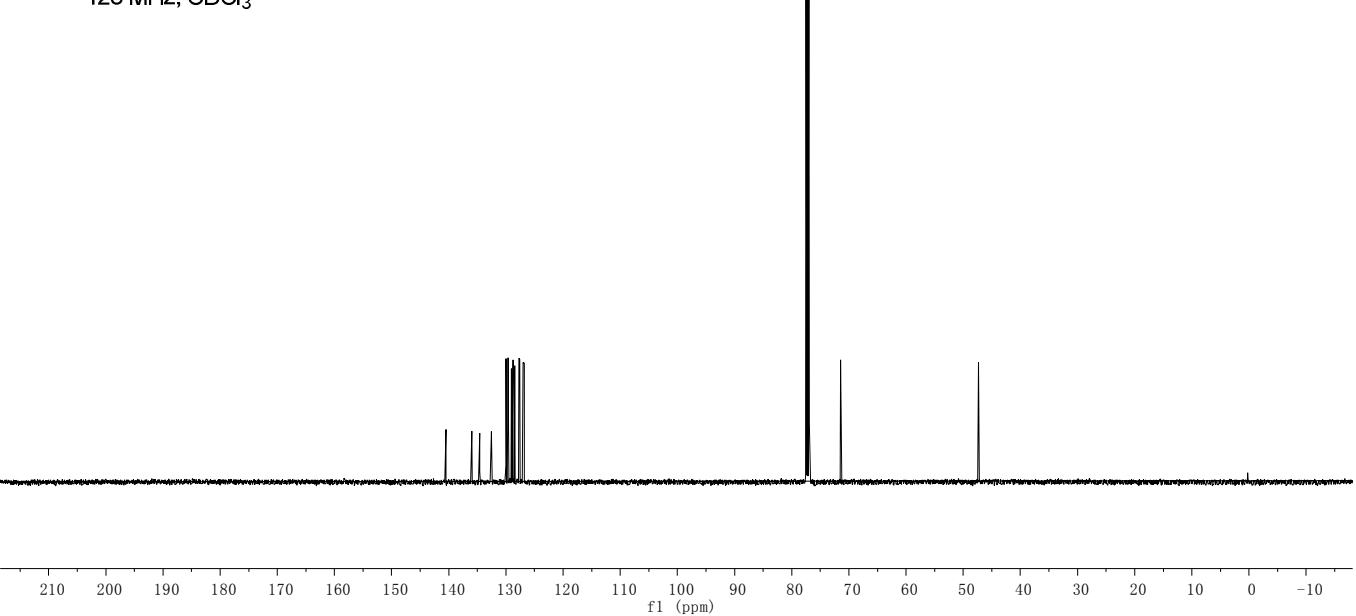

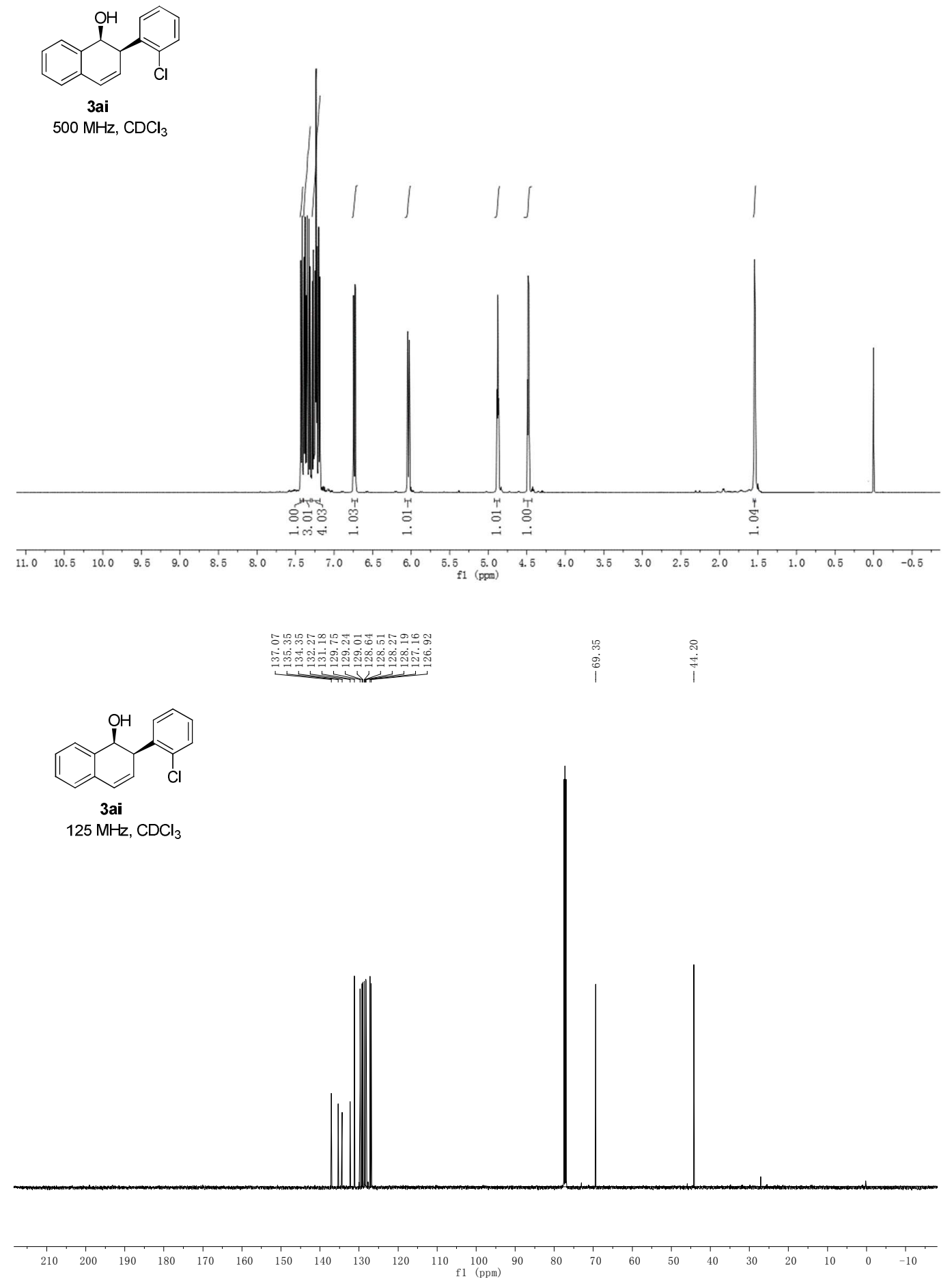

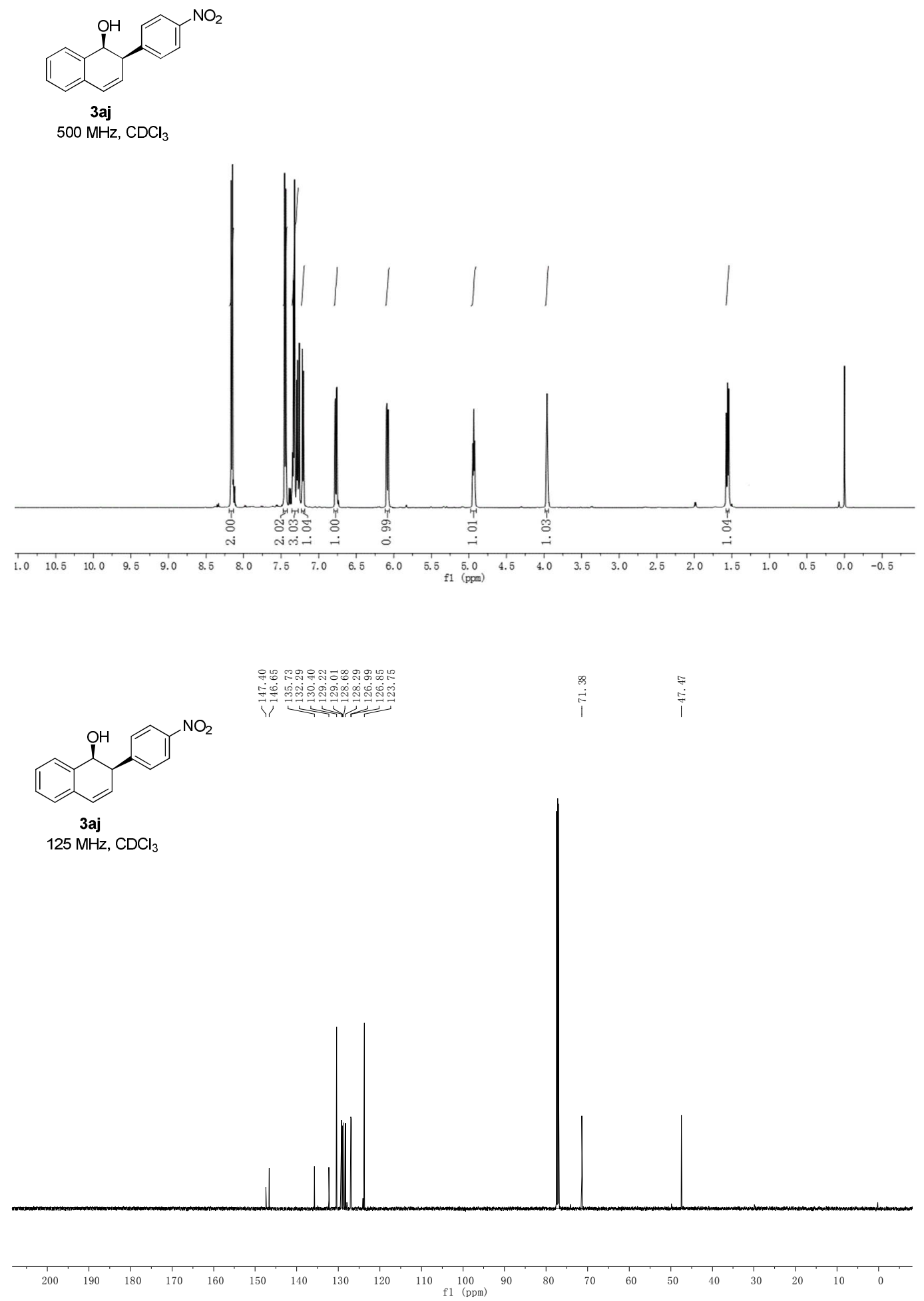

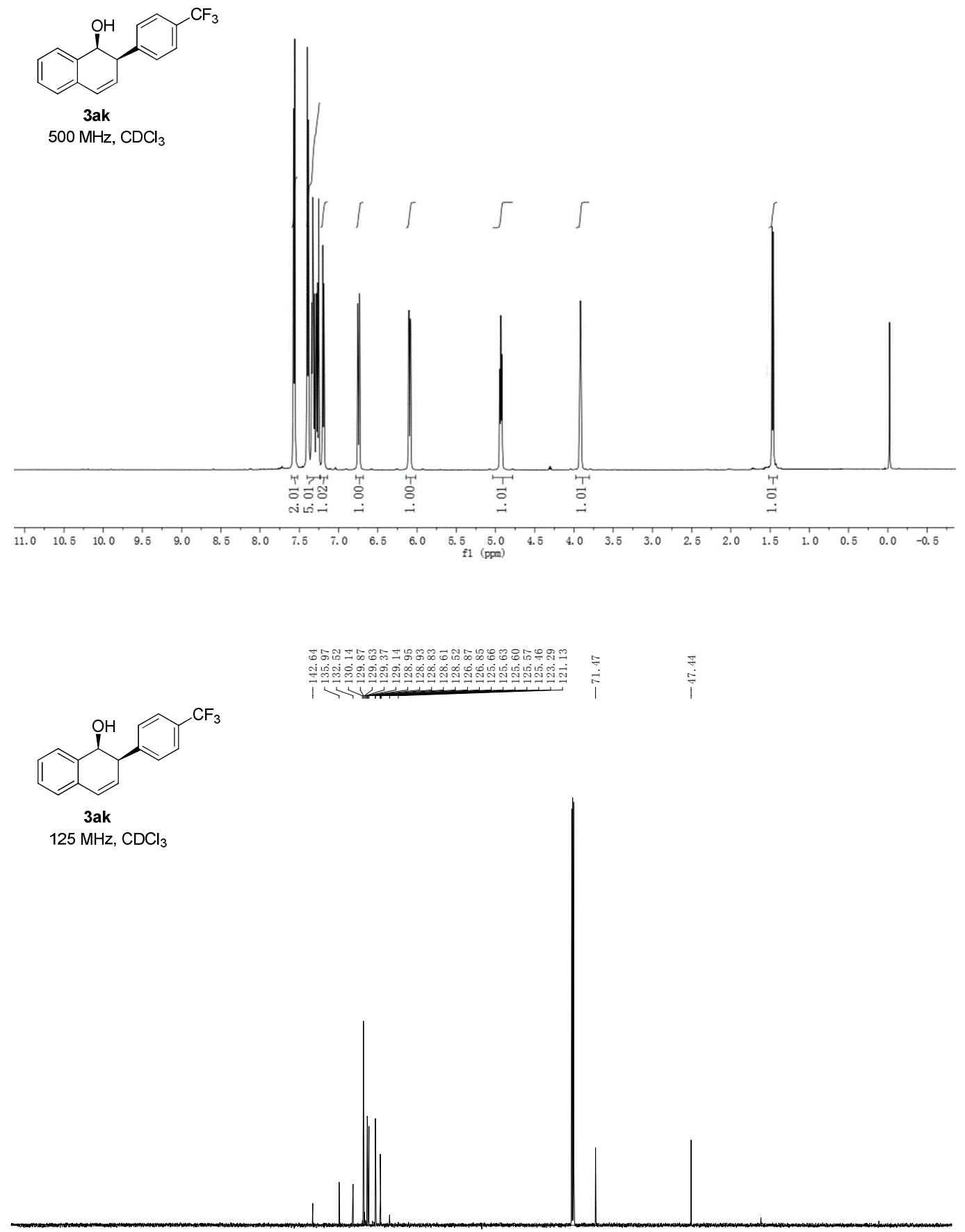

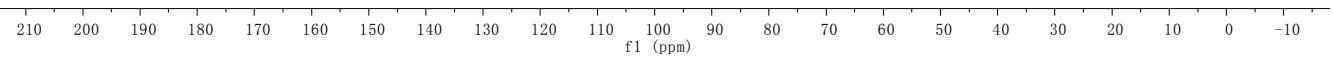




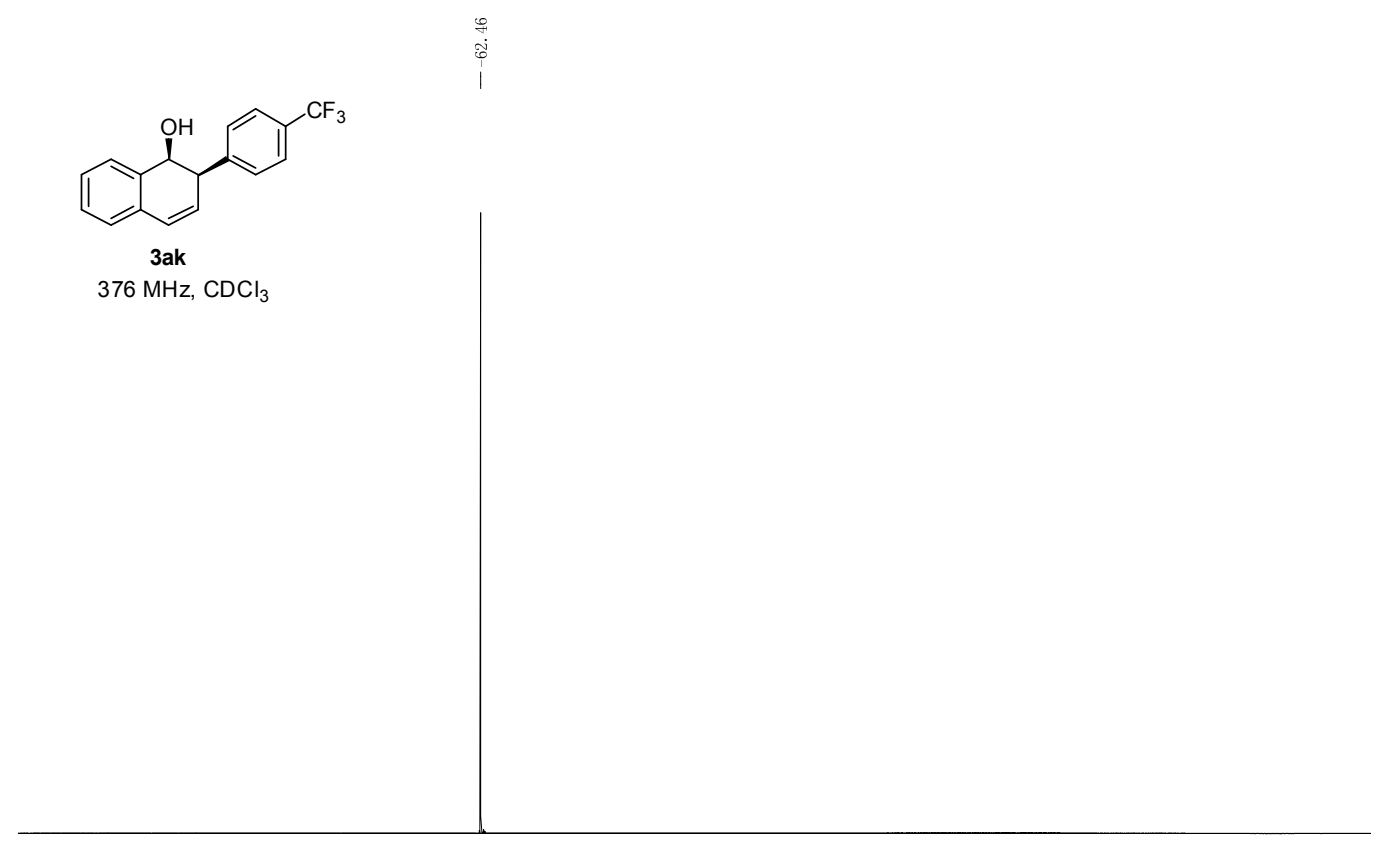

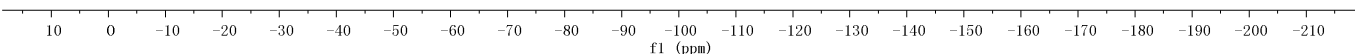



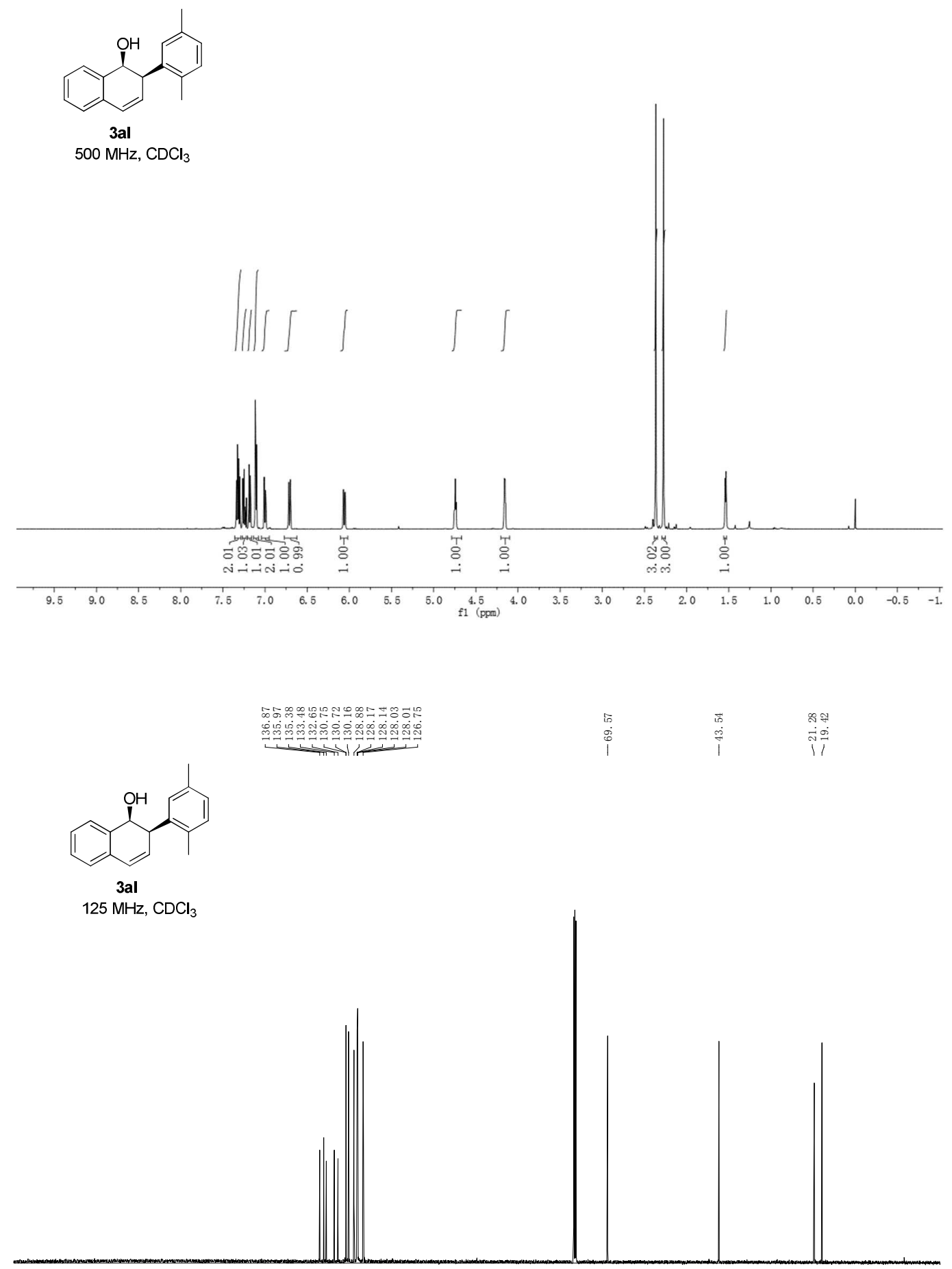


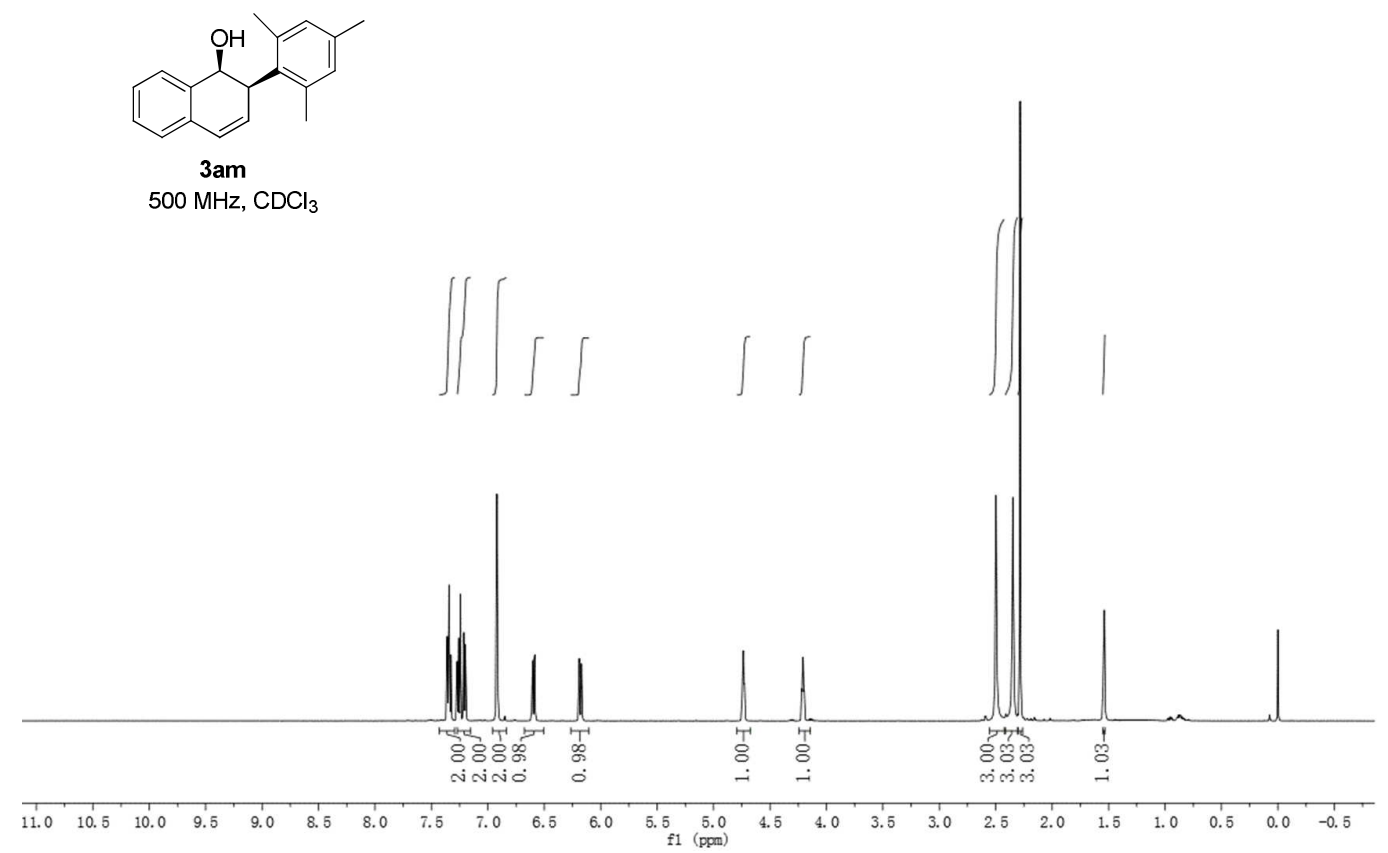

ลㄱํำ约8

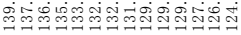

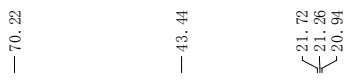

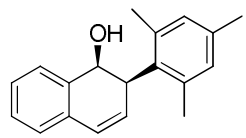

3 am

$125 \mathrm{MHz}, \mathrm{CDCl}_{3}$

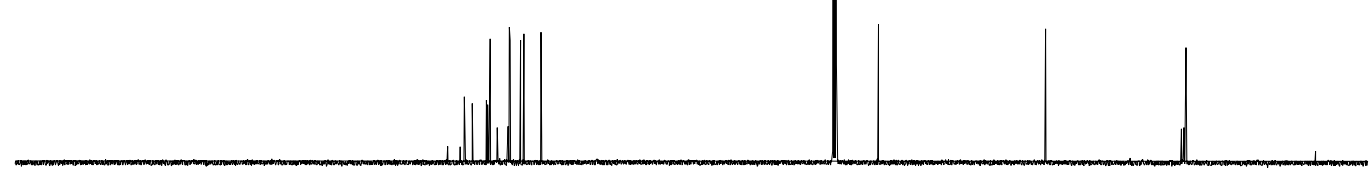

$\begin{array}{llllll}200 & 190 & 180 & 170 & 160 & 150\end{array}$

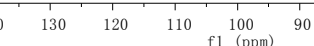



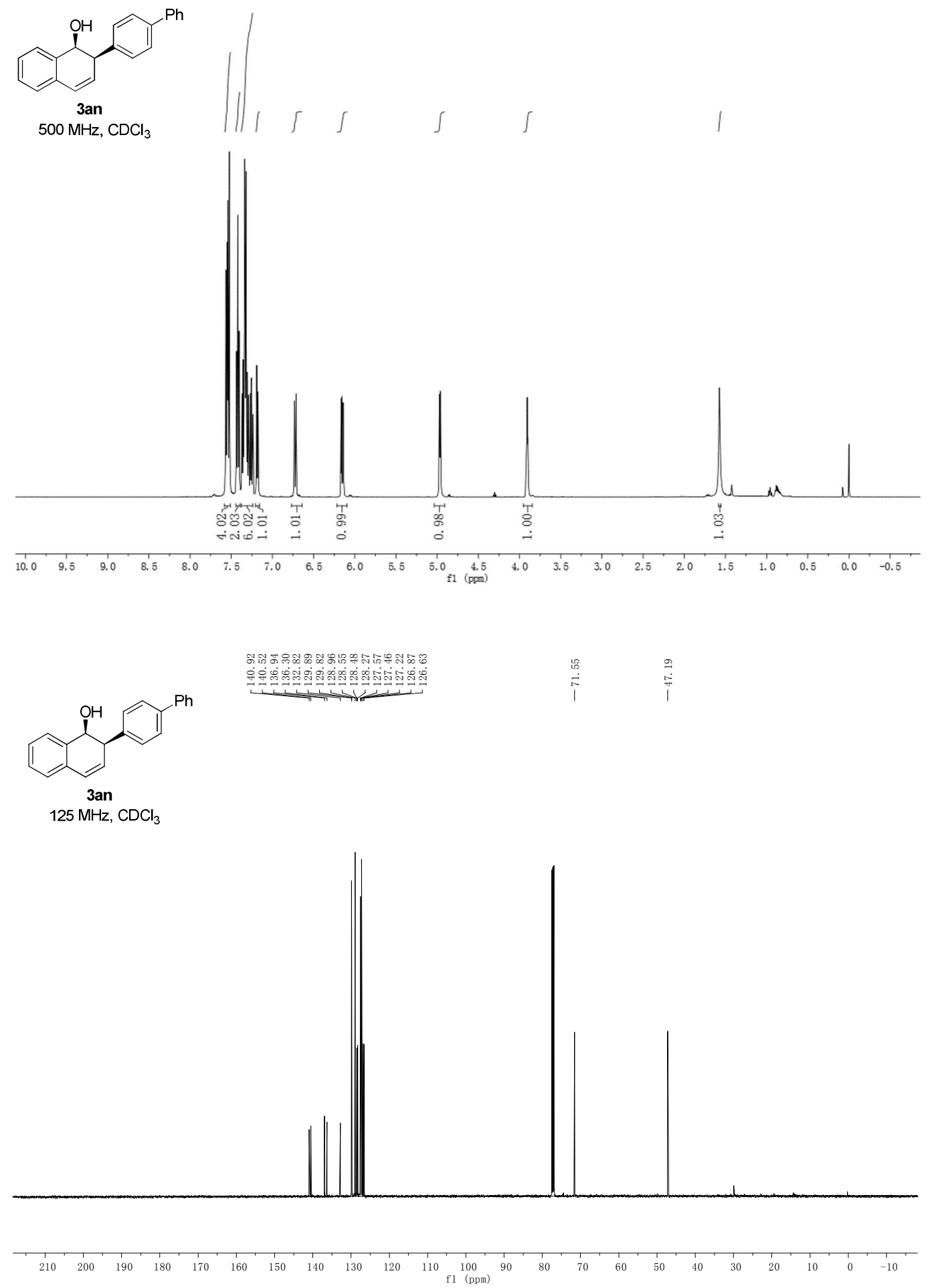


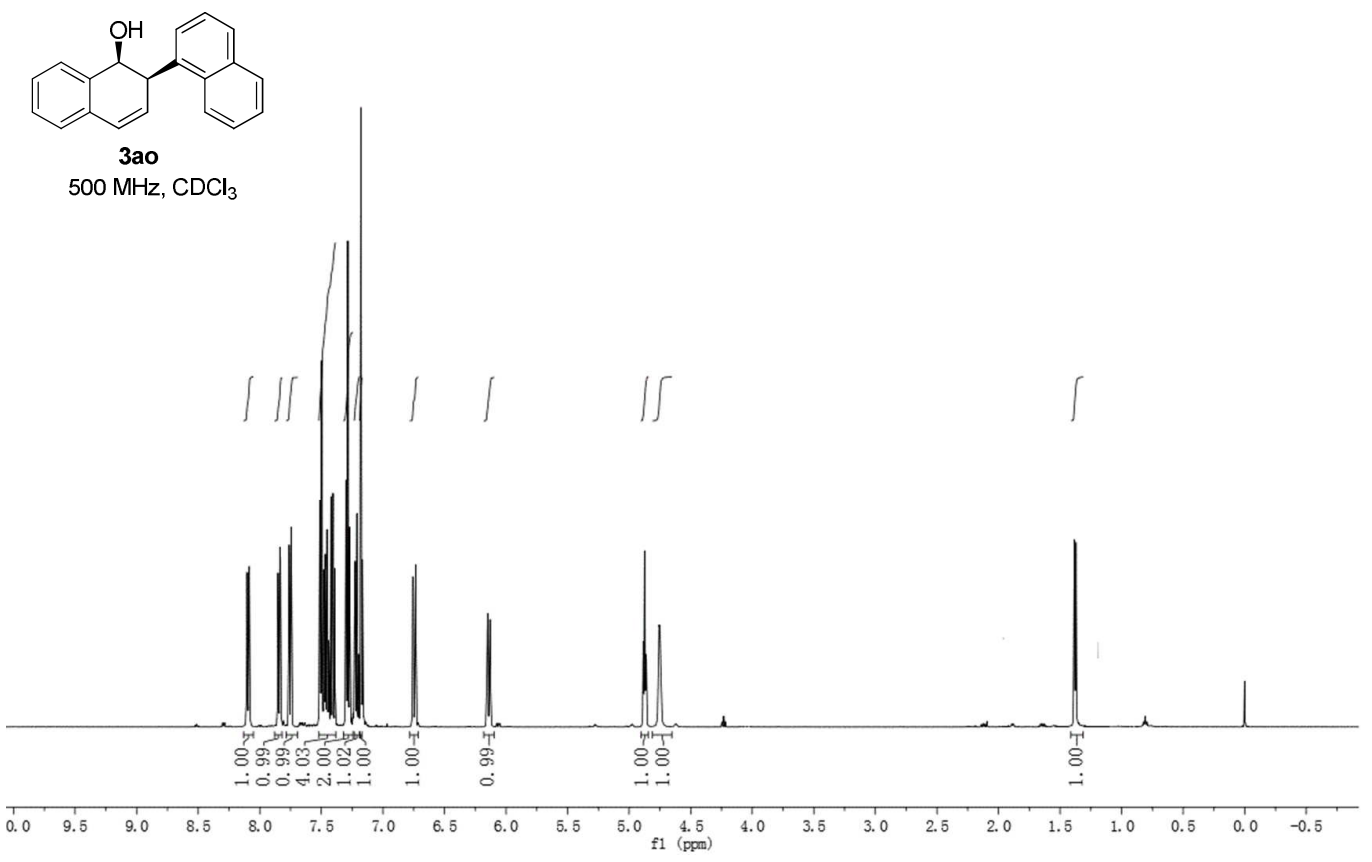

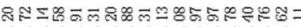

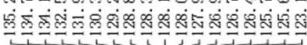
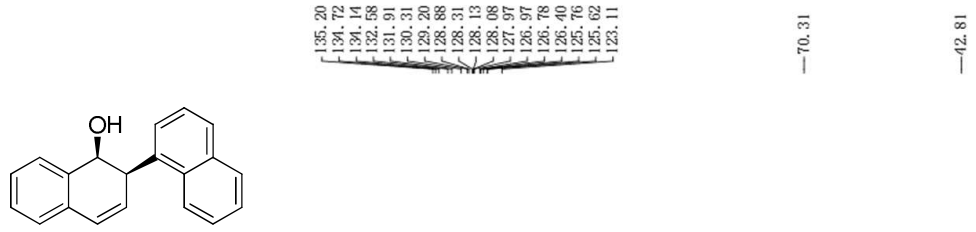

3 ao

$125 \mathrm{MHz}, \mathrm{CDCl}_{3}$
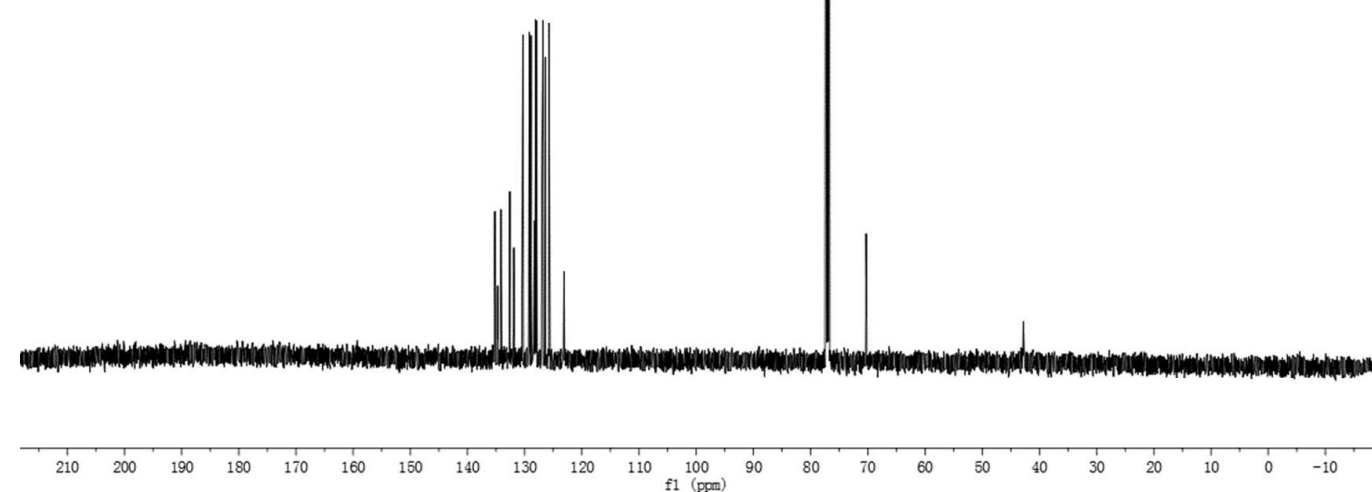


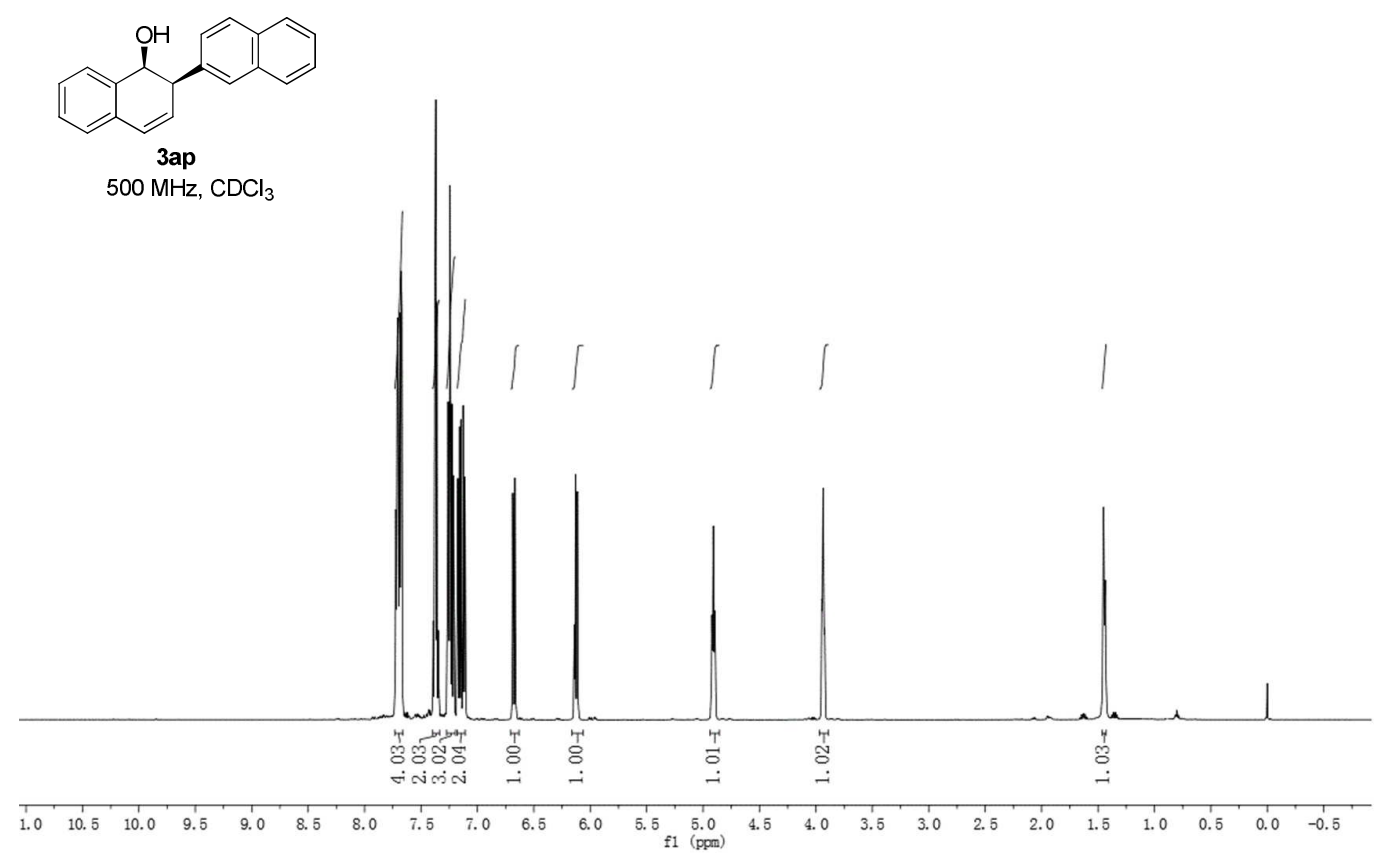

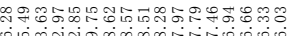

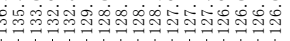
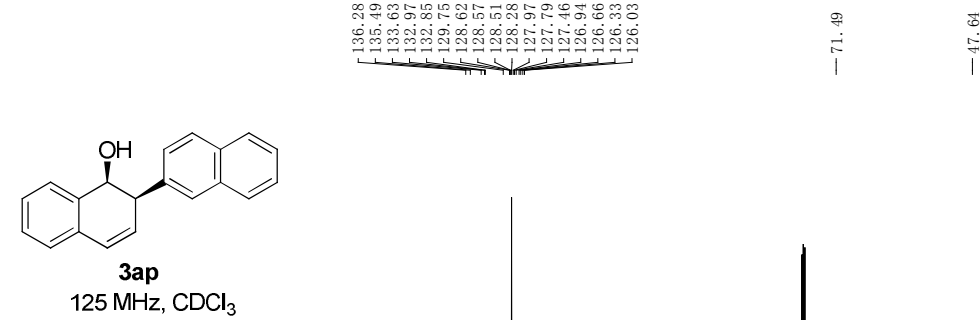

$5 \mathrm{MHz}, \mathrm{CDCl}_{3}$
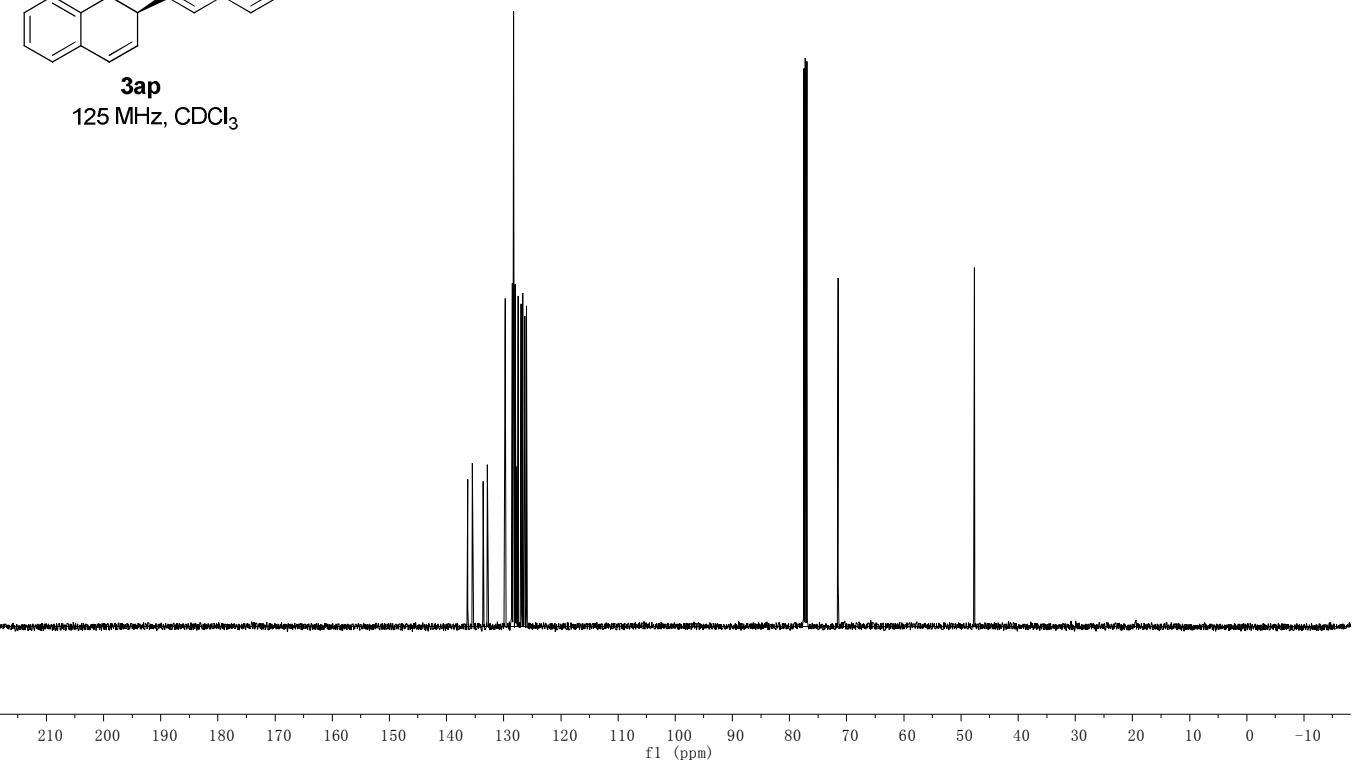

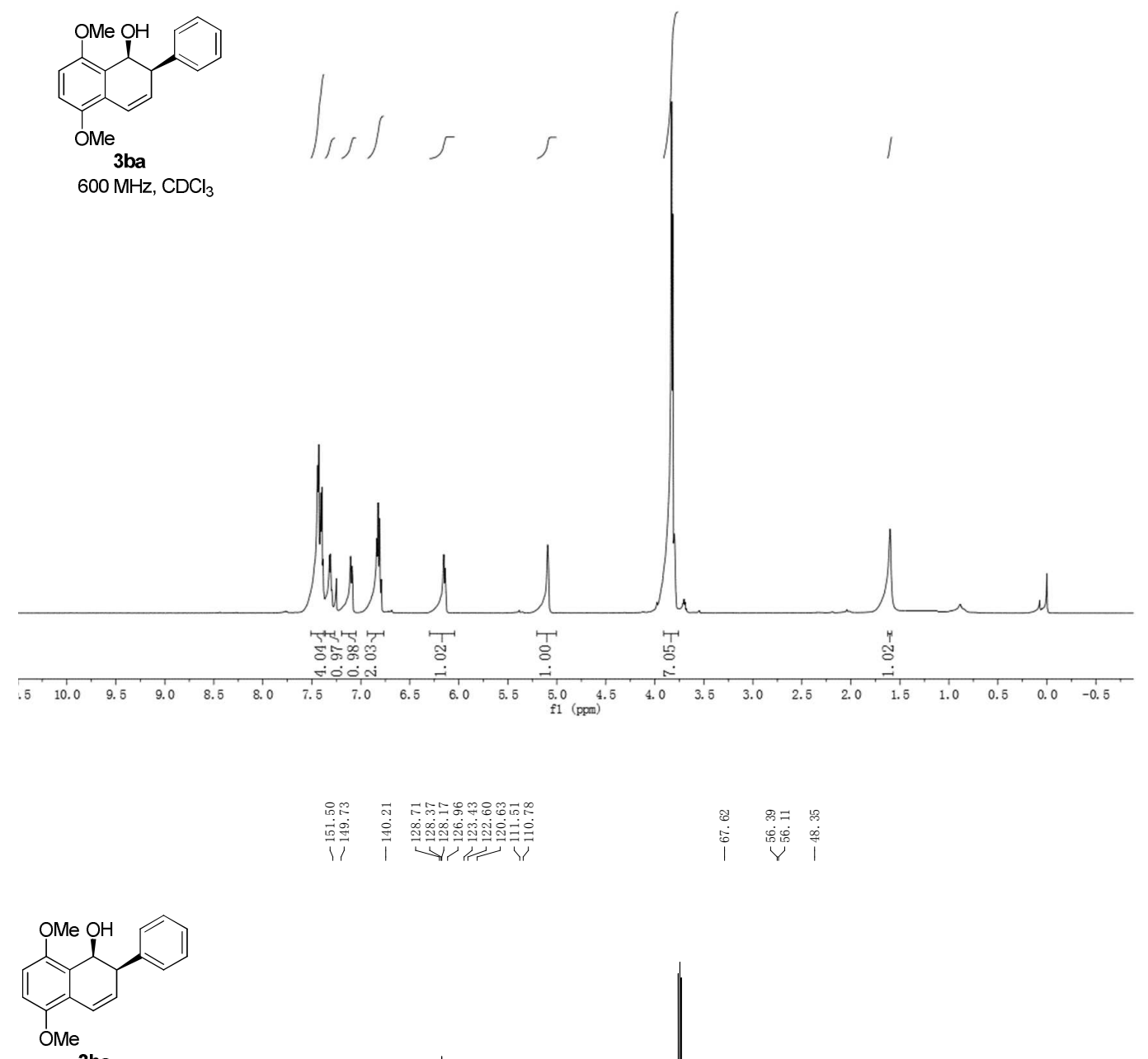

3 ba

$100 \mathrm{MHz} \mathrm{CDCl}_{3}$
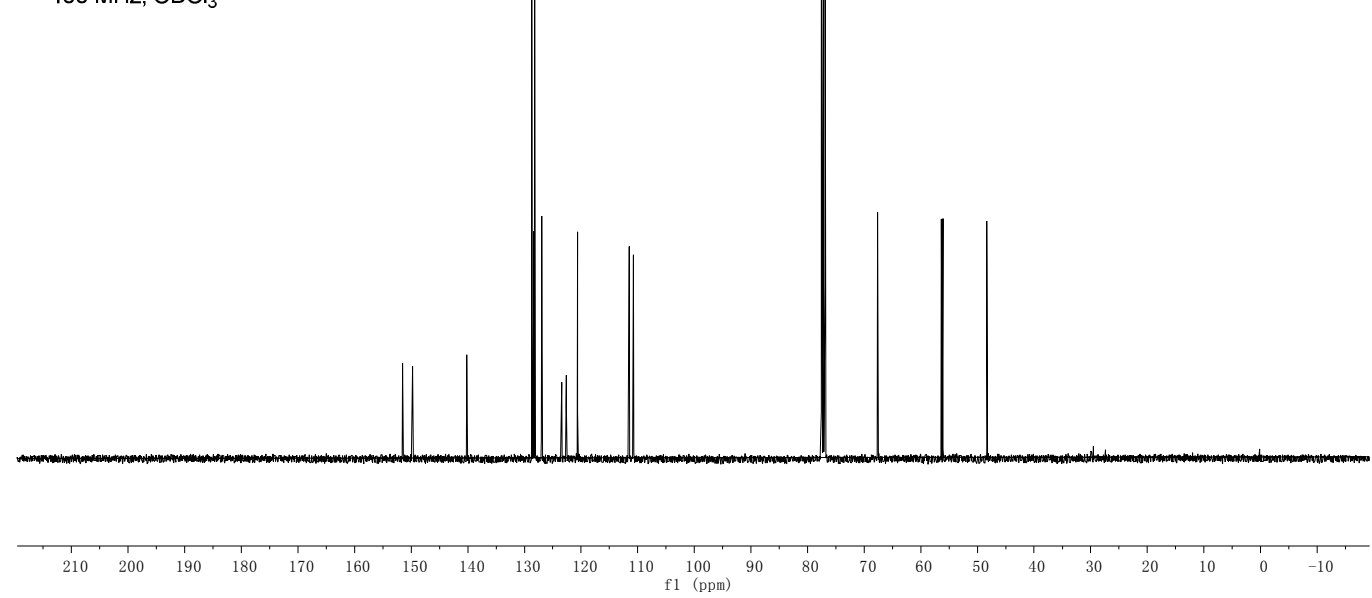

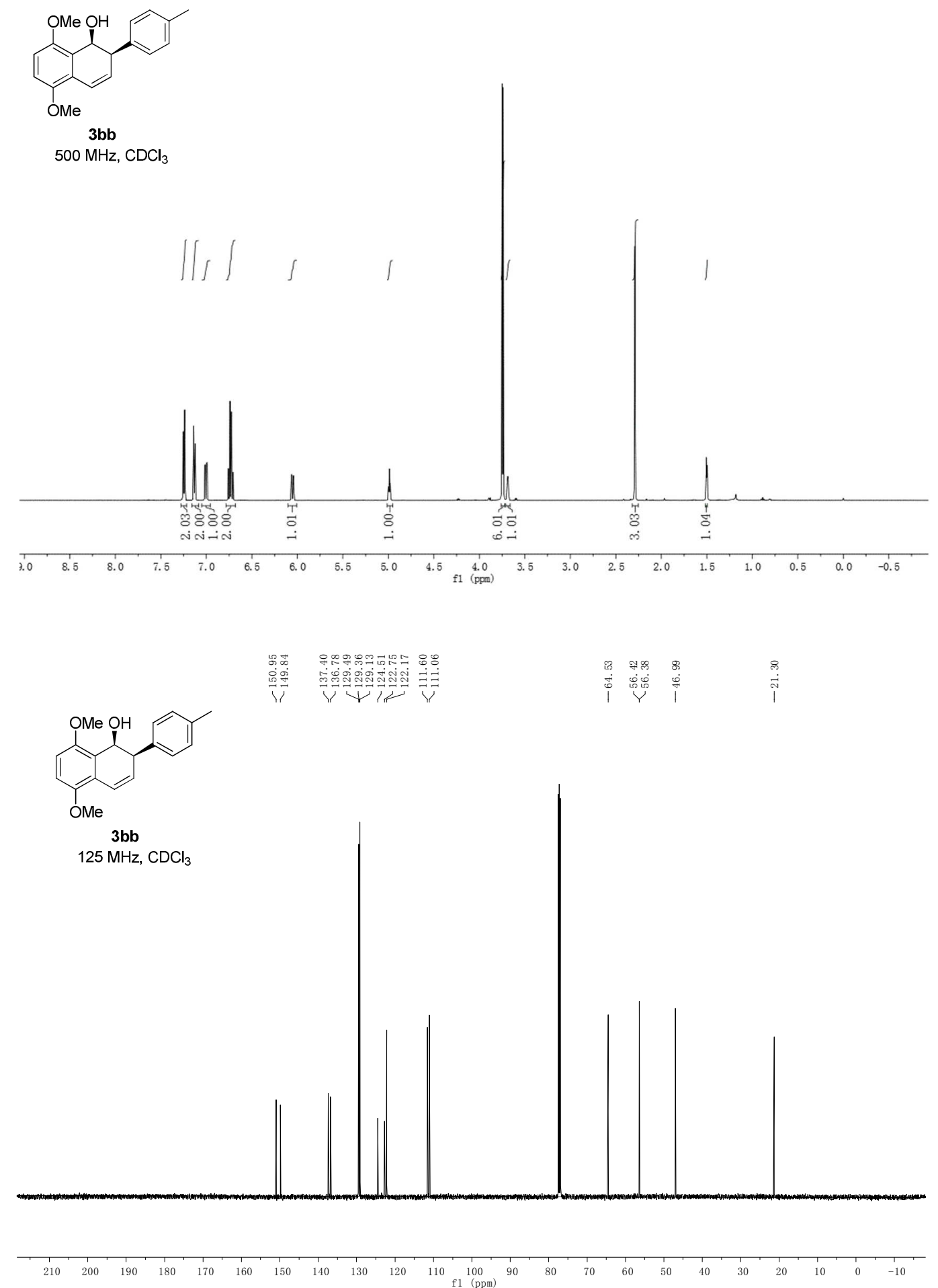

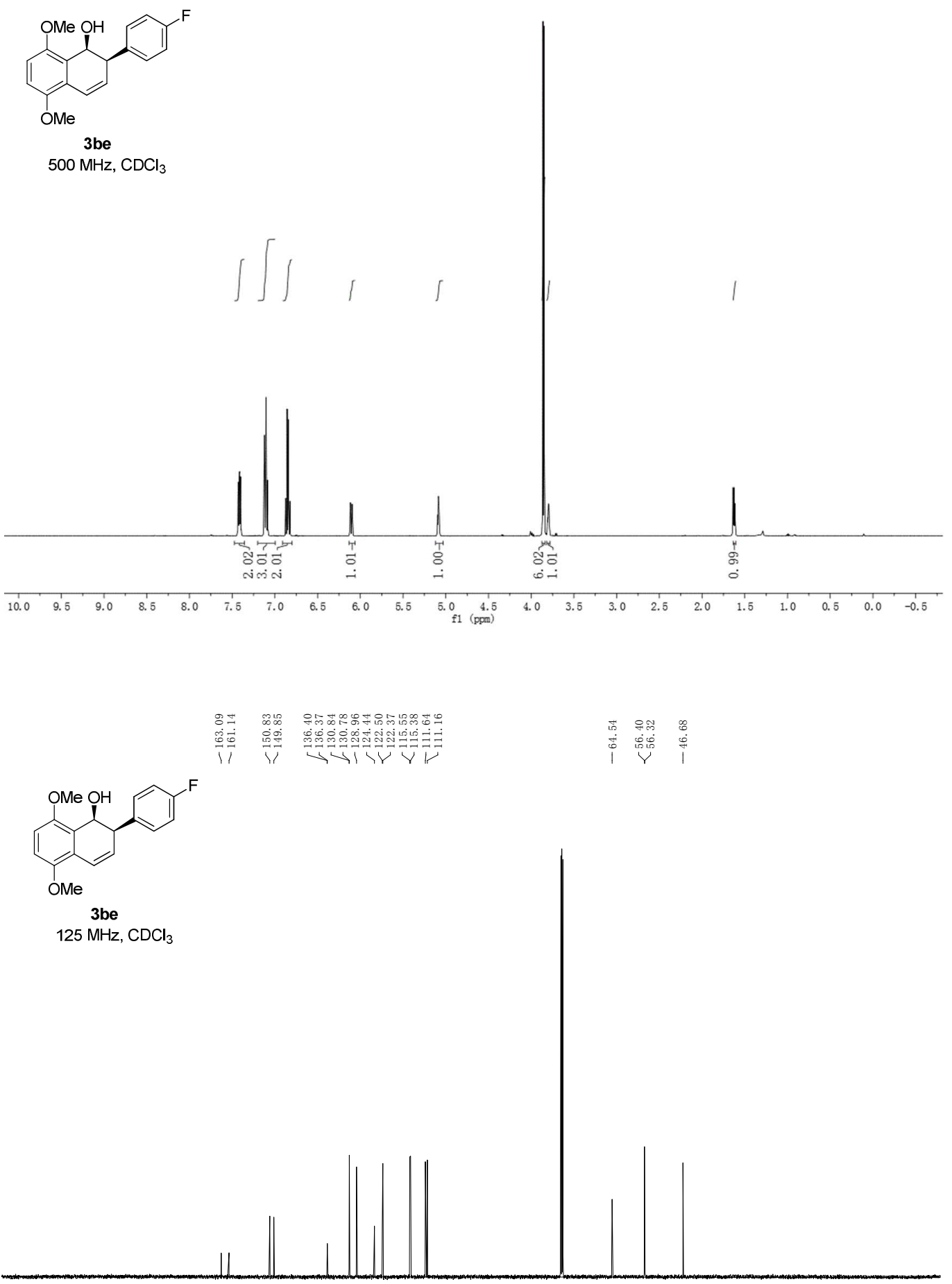

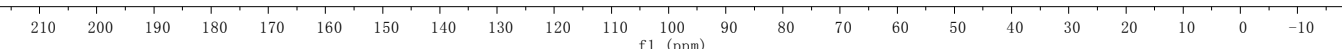




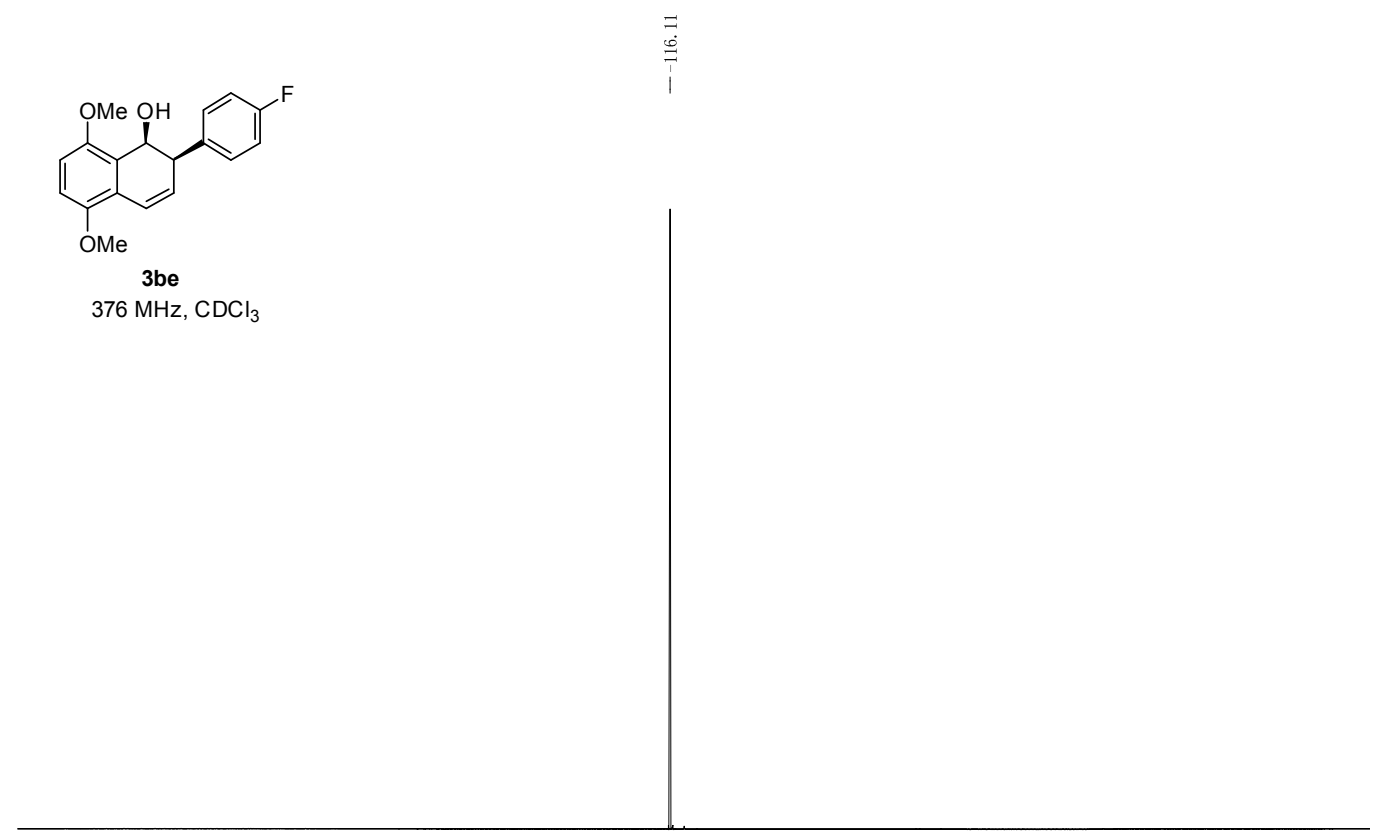

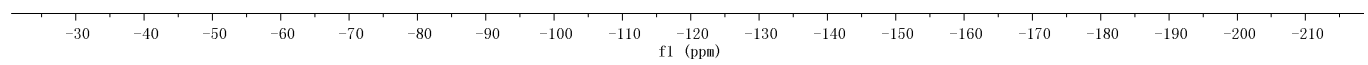



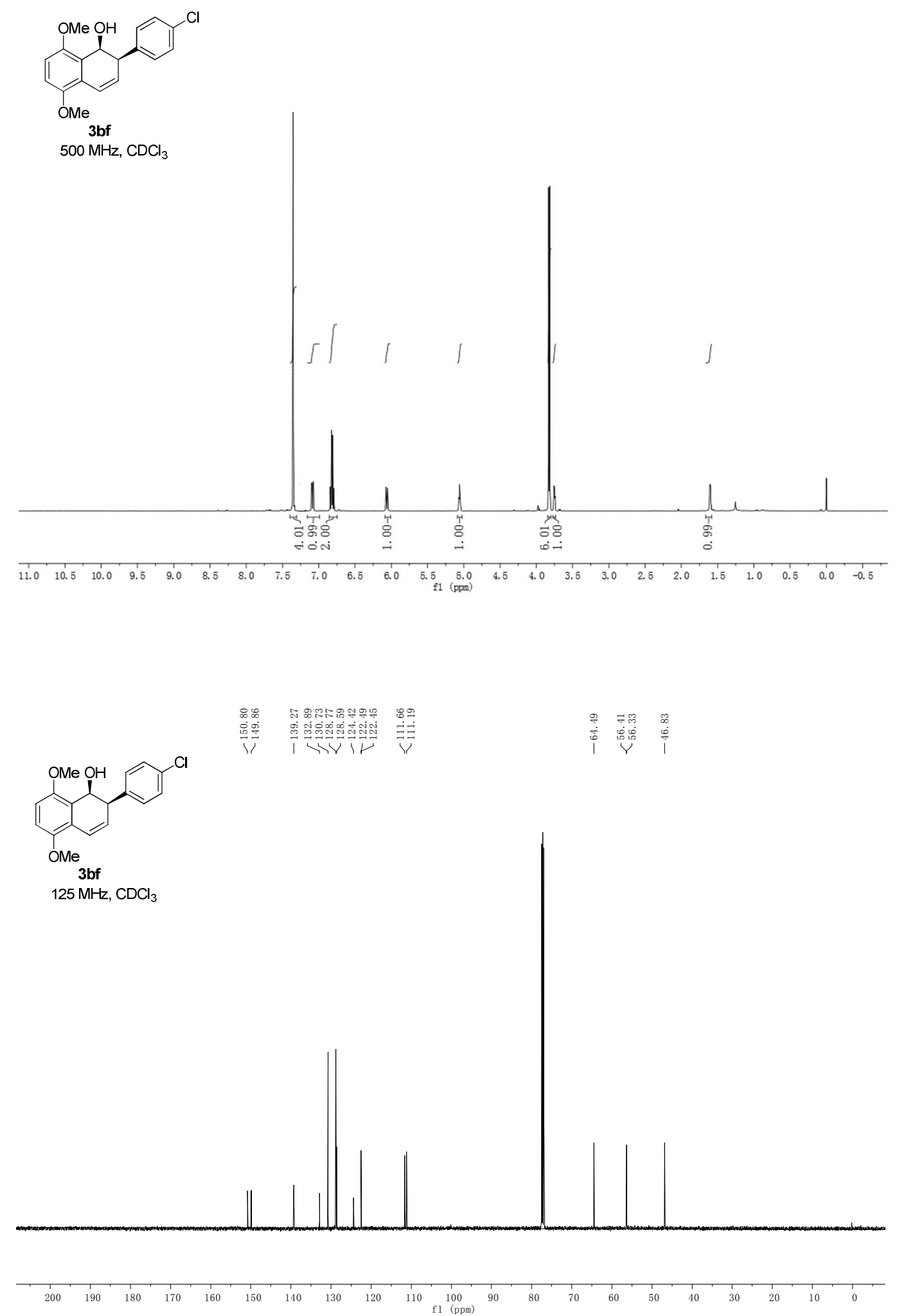


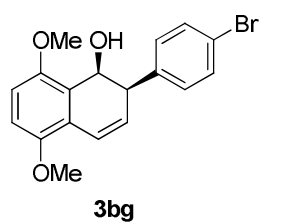

$500 \mathrm{MHz}, \mathrm{CDCl}_{3}$
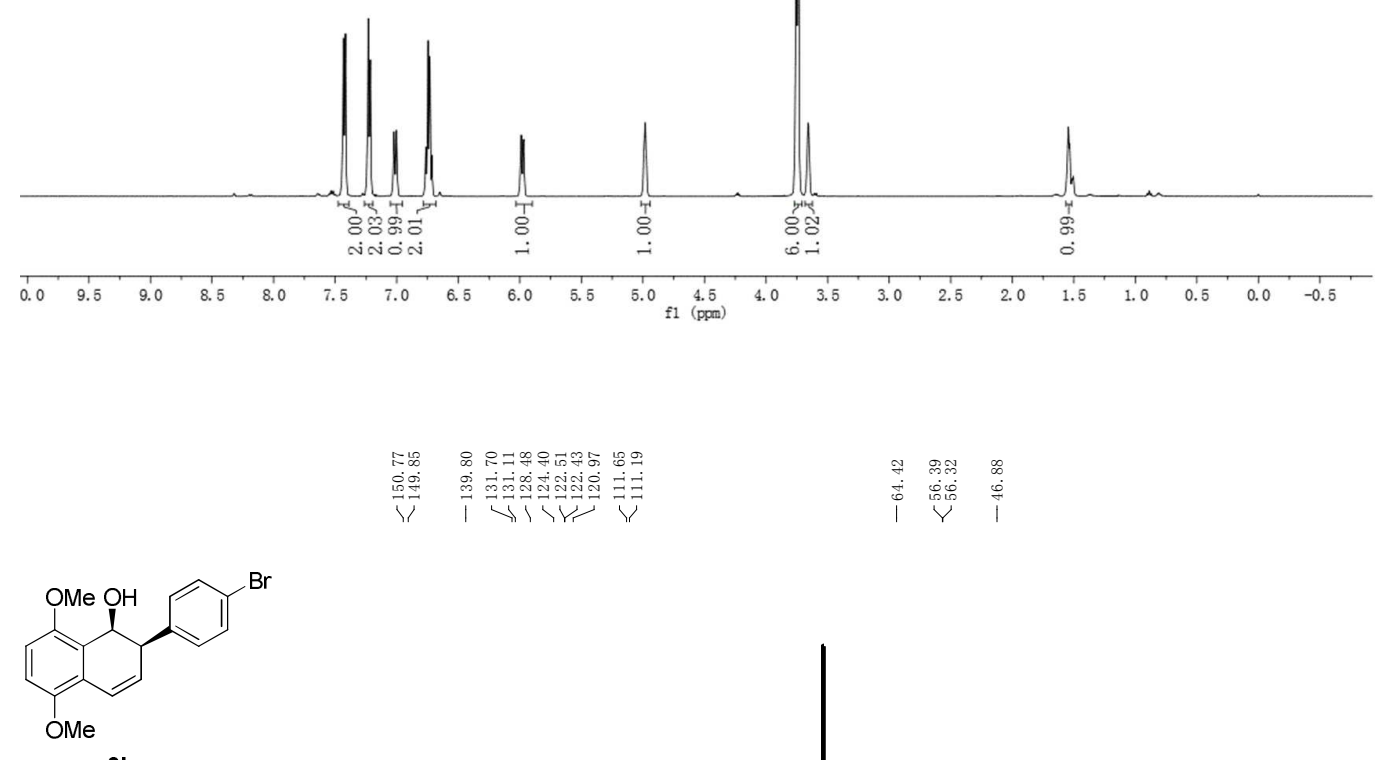

$3 \mathrm{bg}$

$125 \mathrm{MHz}, \mathrm{CDCl}_{3}$

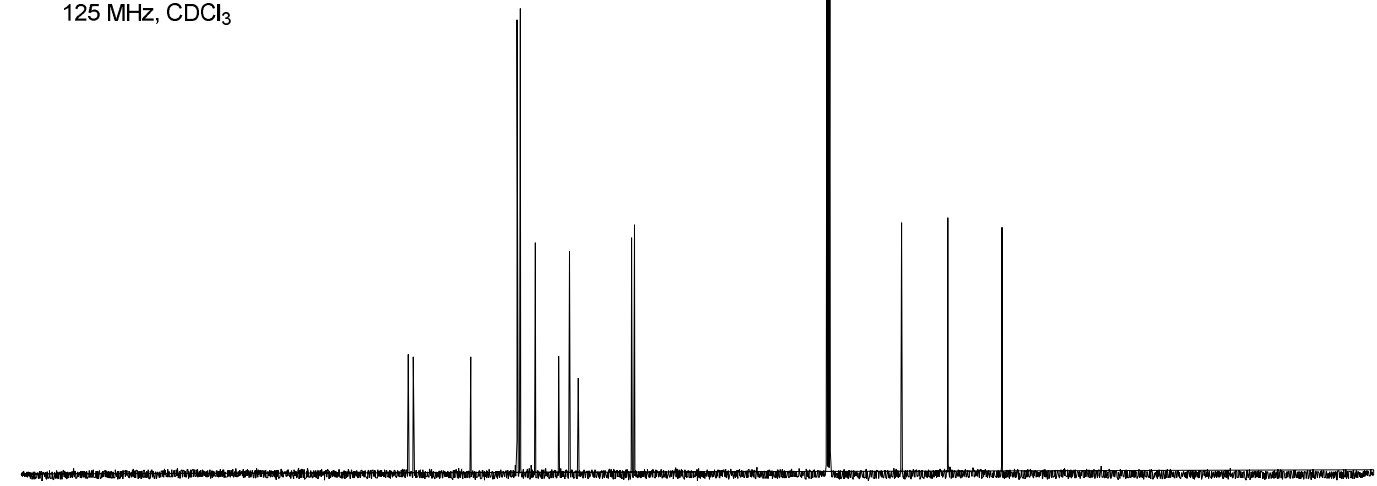

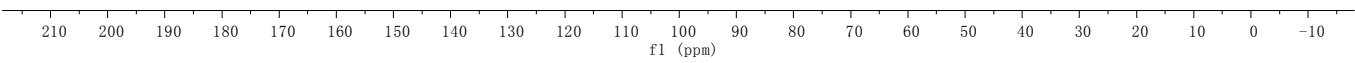



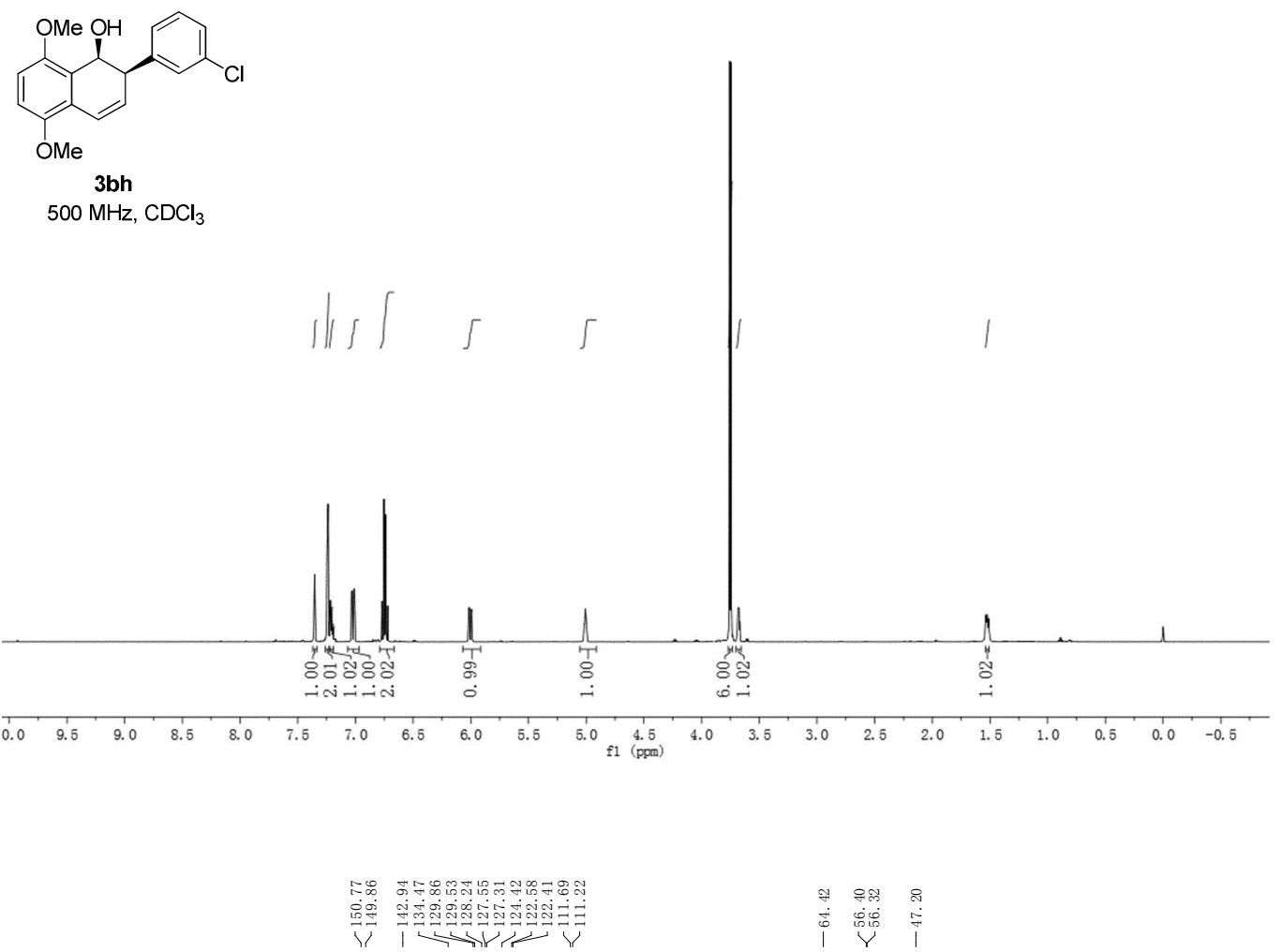

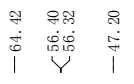

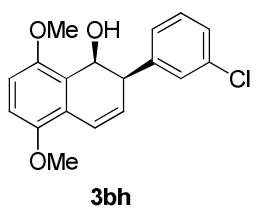

$125 \mathrm{MHz} \mathrm{CDCl}_{3}$
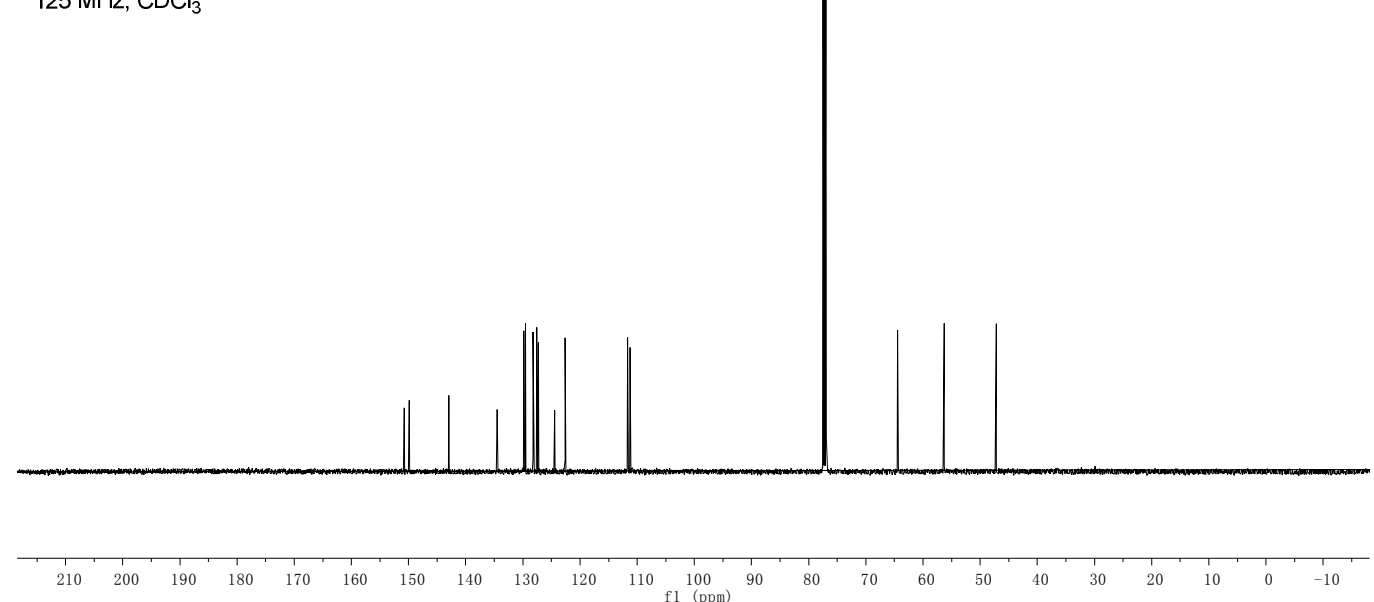


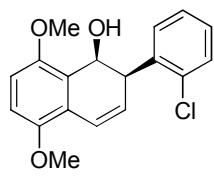

$3 \mathbf{b i}$

$500 \mathrm{MHz}, \mathrm{CDCl}_{3}$
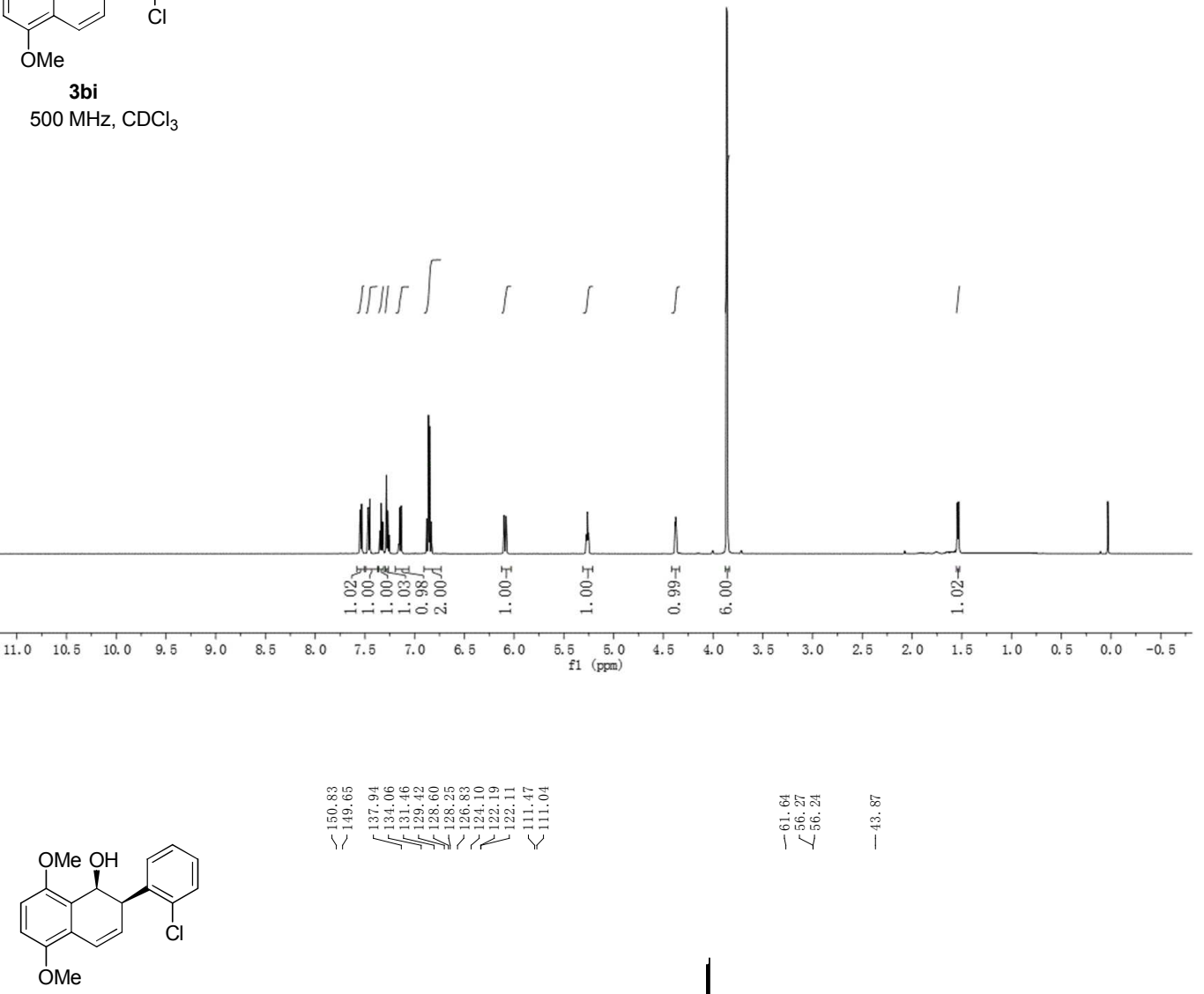

๓ 富

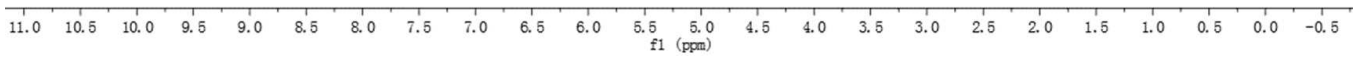

$3 \mathrm{bi}$

$125 \mathrm{MHz} \mathrm{CDCl}_{3}$

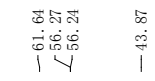
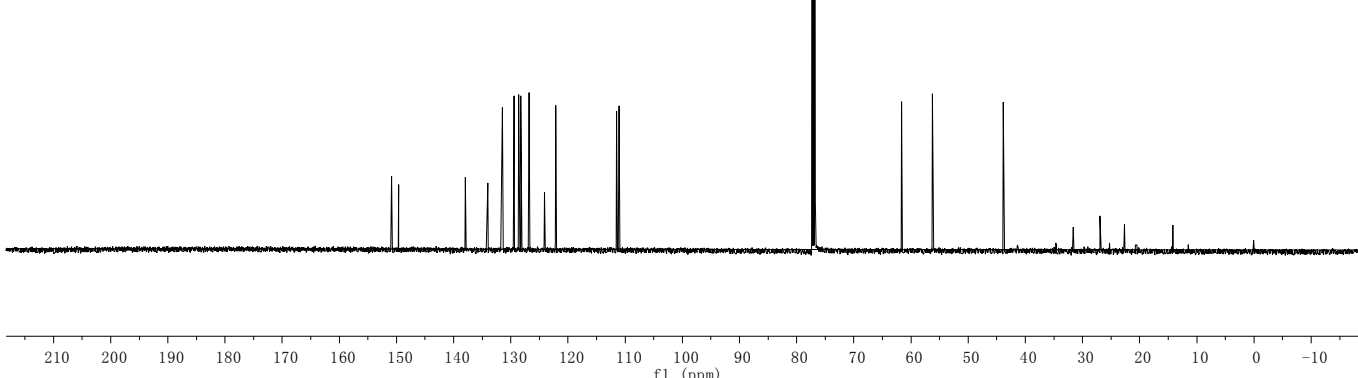


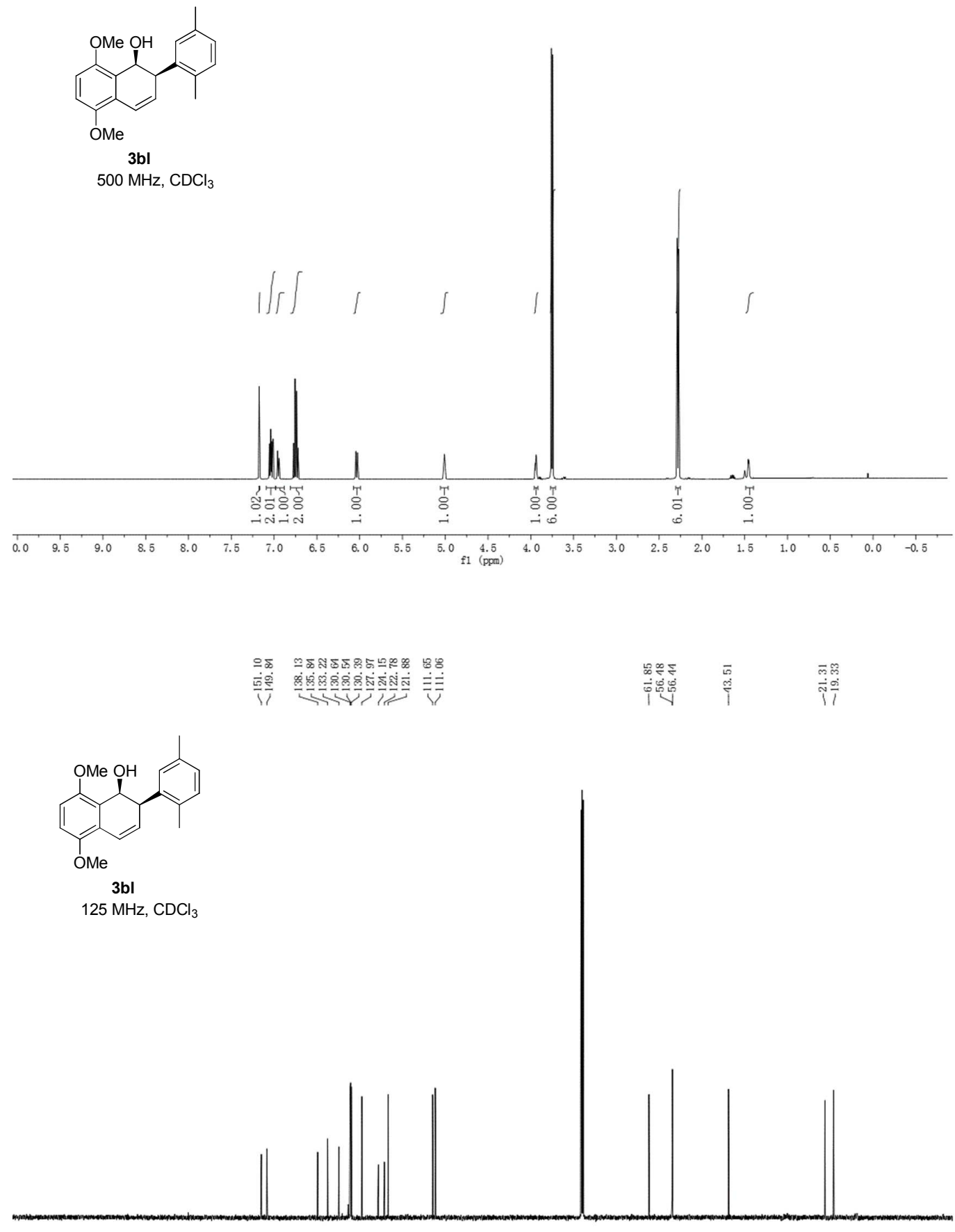

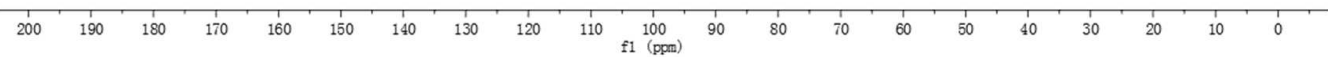




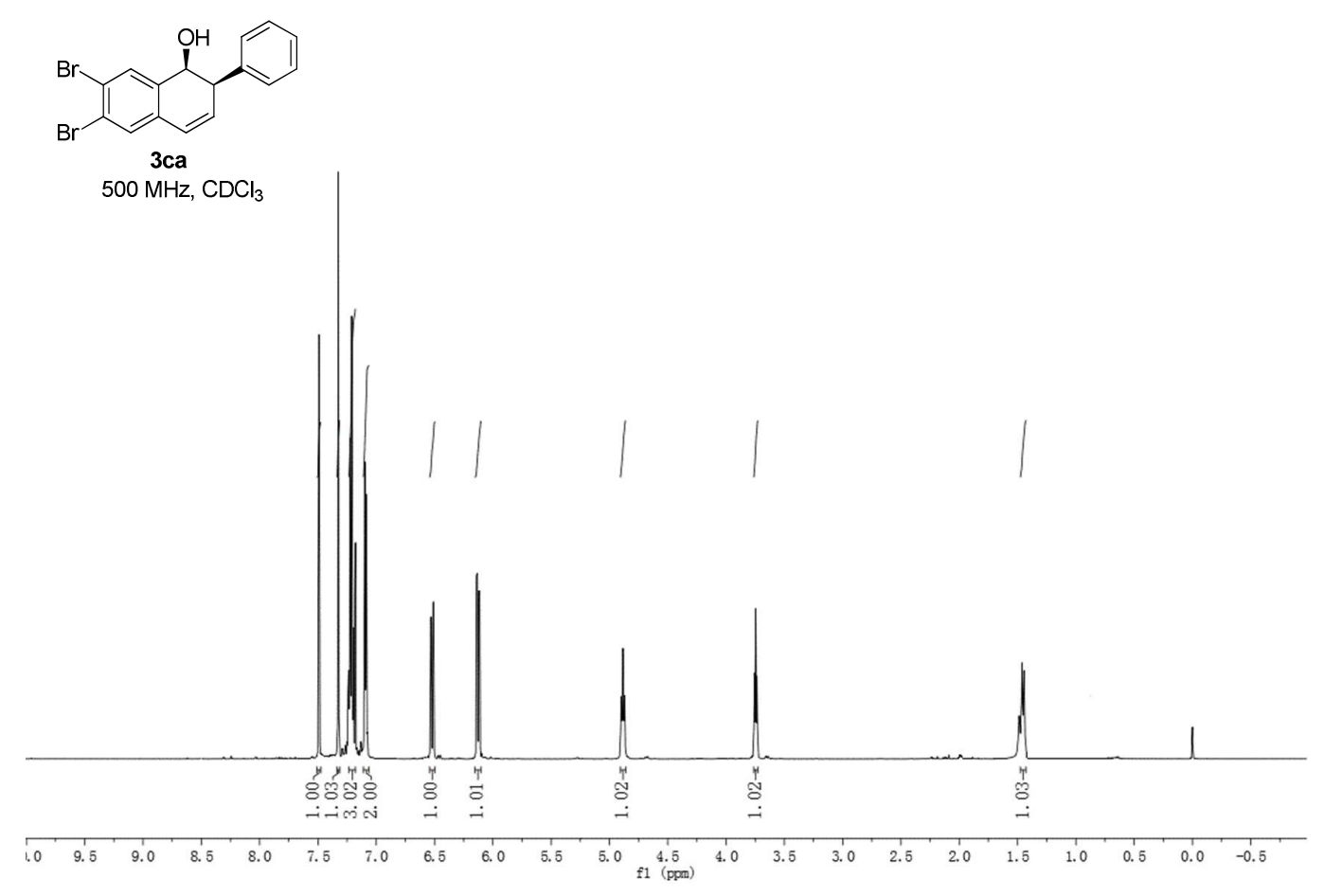

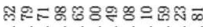

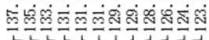

$\stackrel{5}{1}$
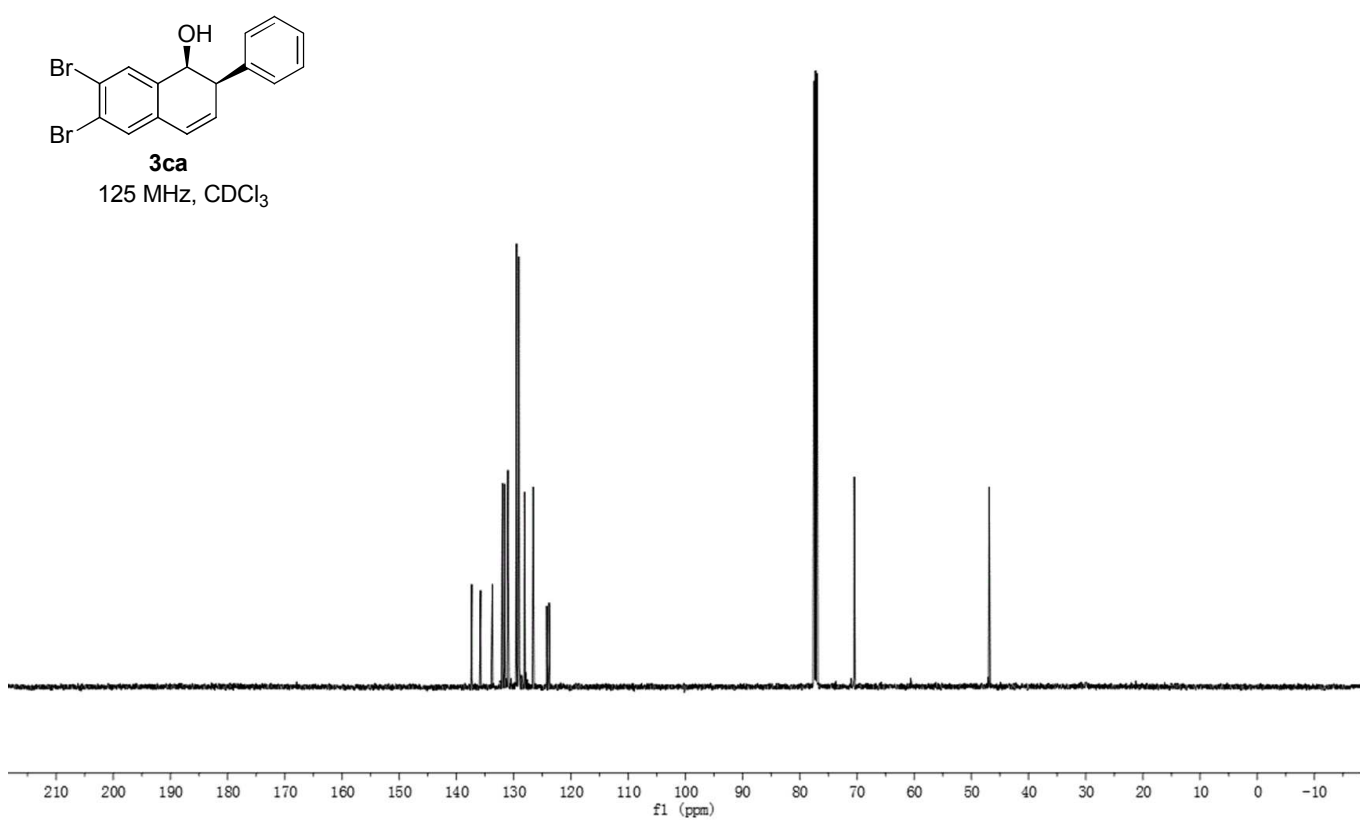


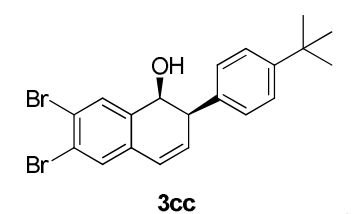

$500 \mathrm{MHz}, \mathrm{CHCl}_{3} \quad j / /, / / 1 /$
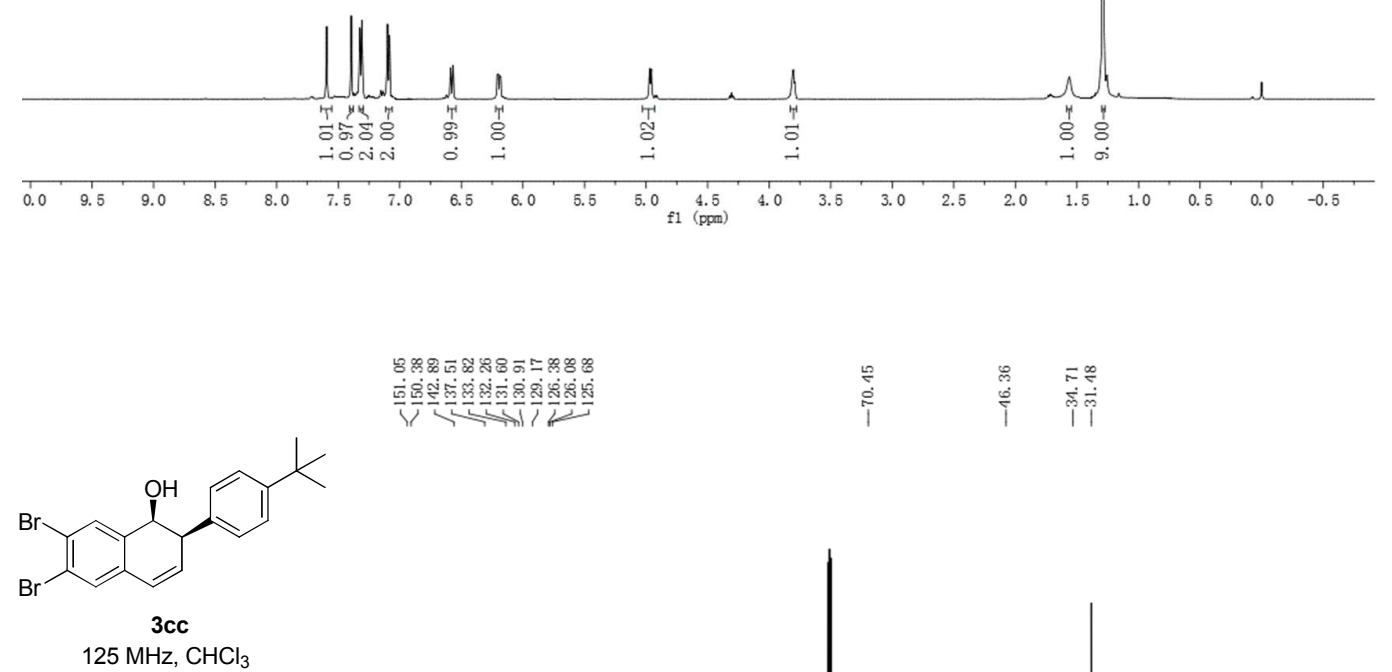

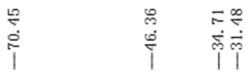
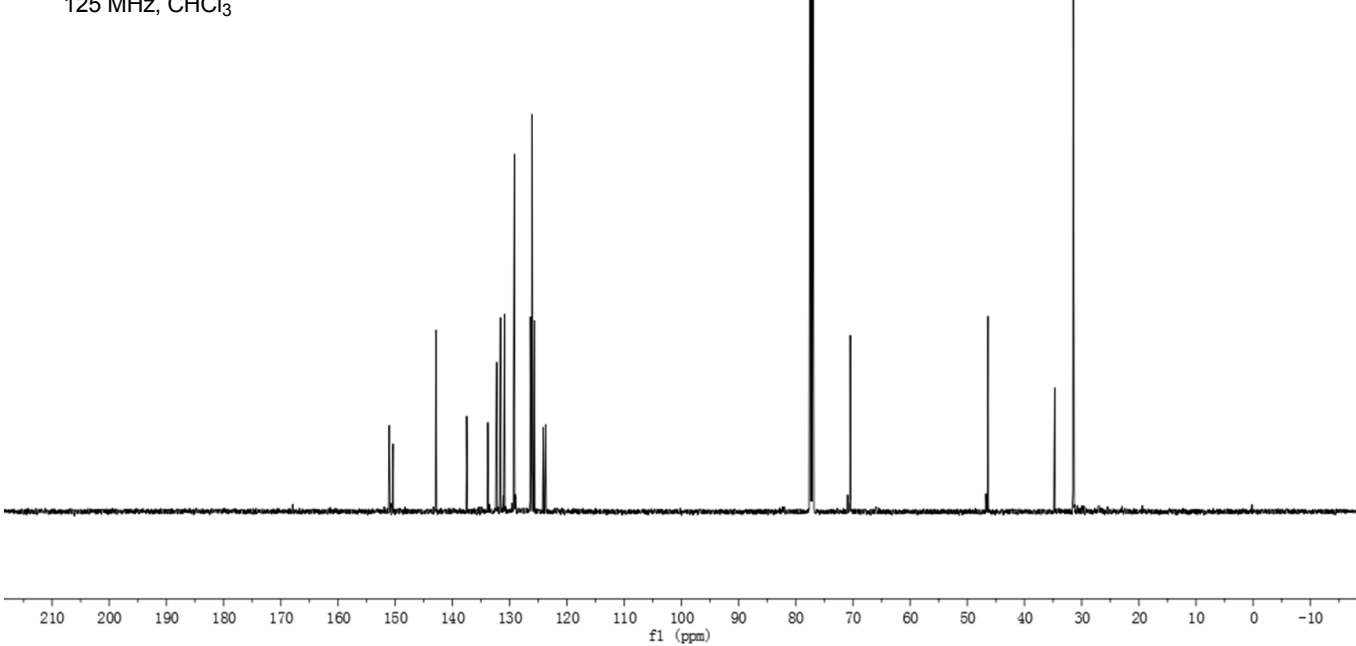

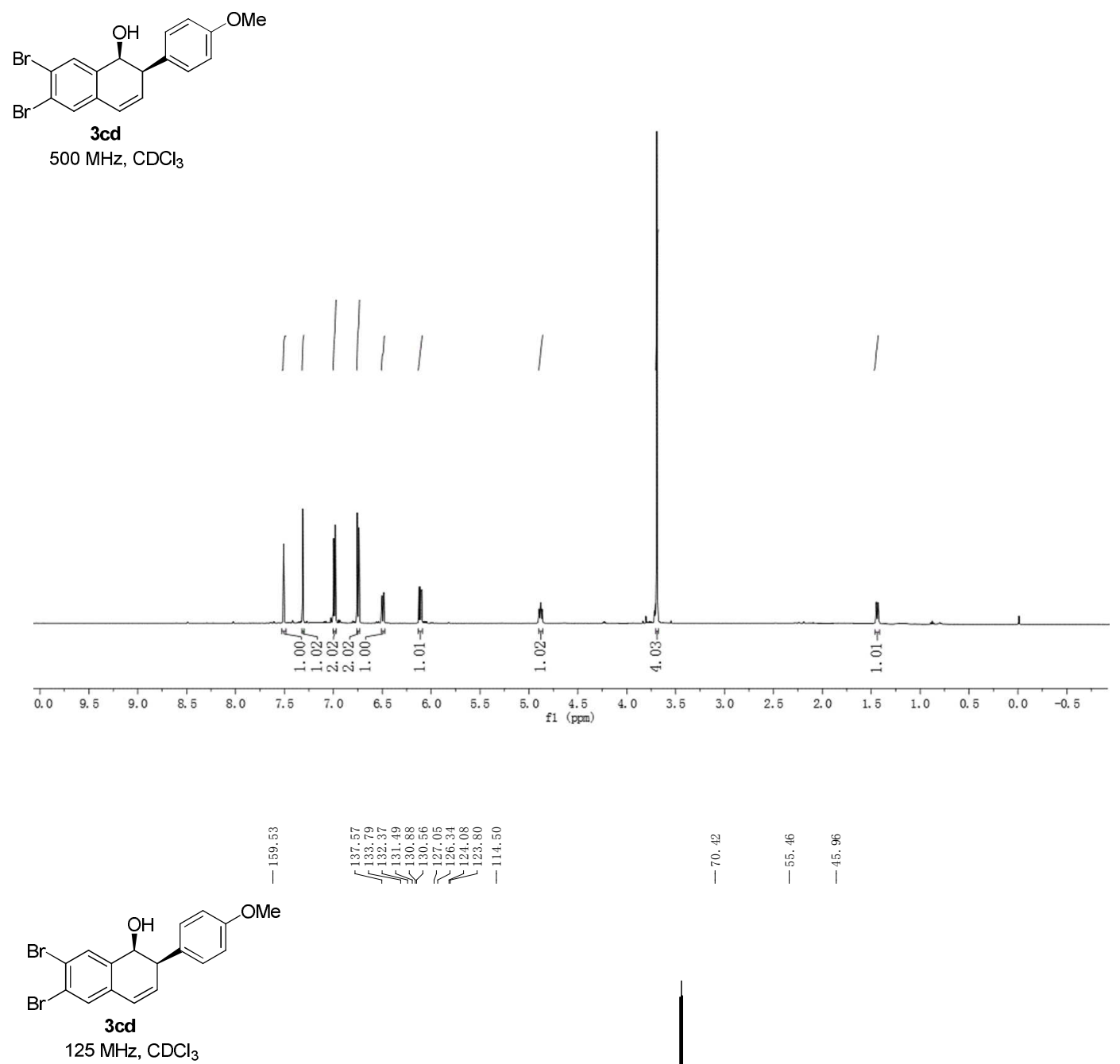

$25 \mathrm{MHz}, \mathrm{CDCl}_{3}$
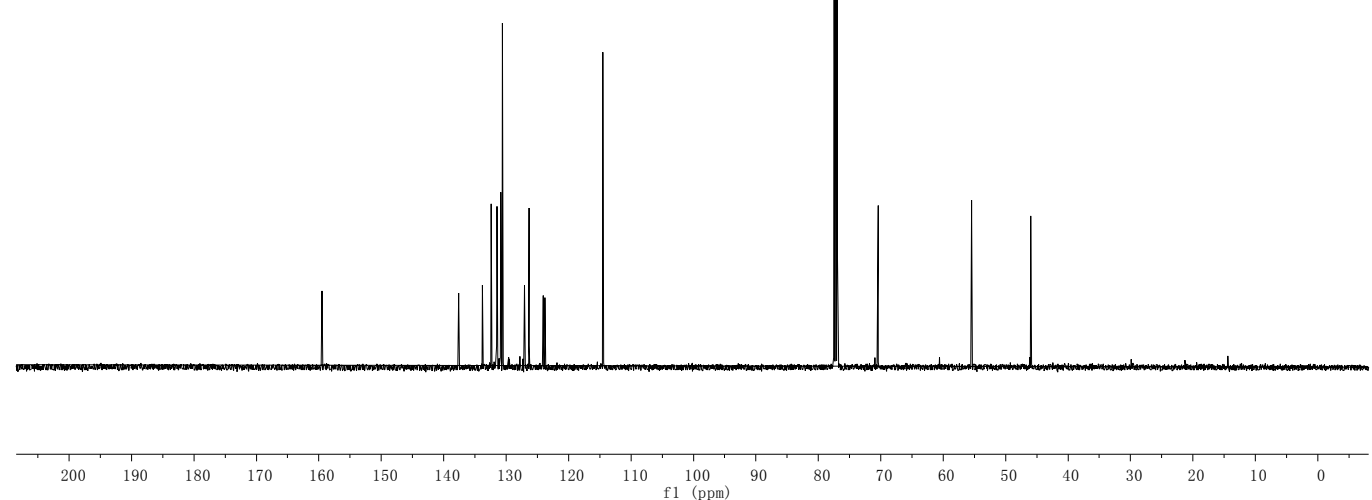

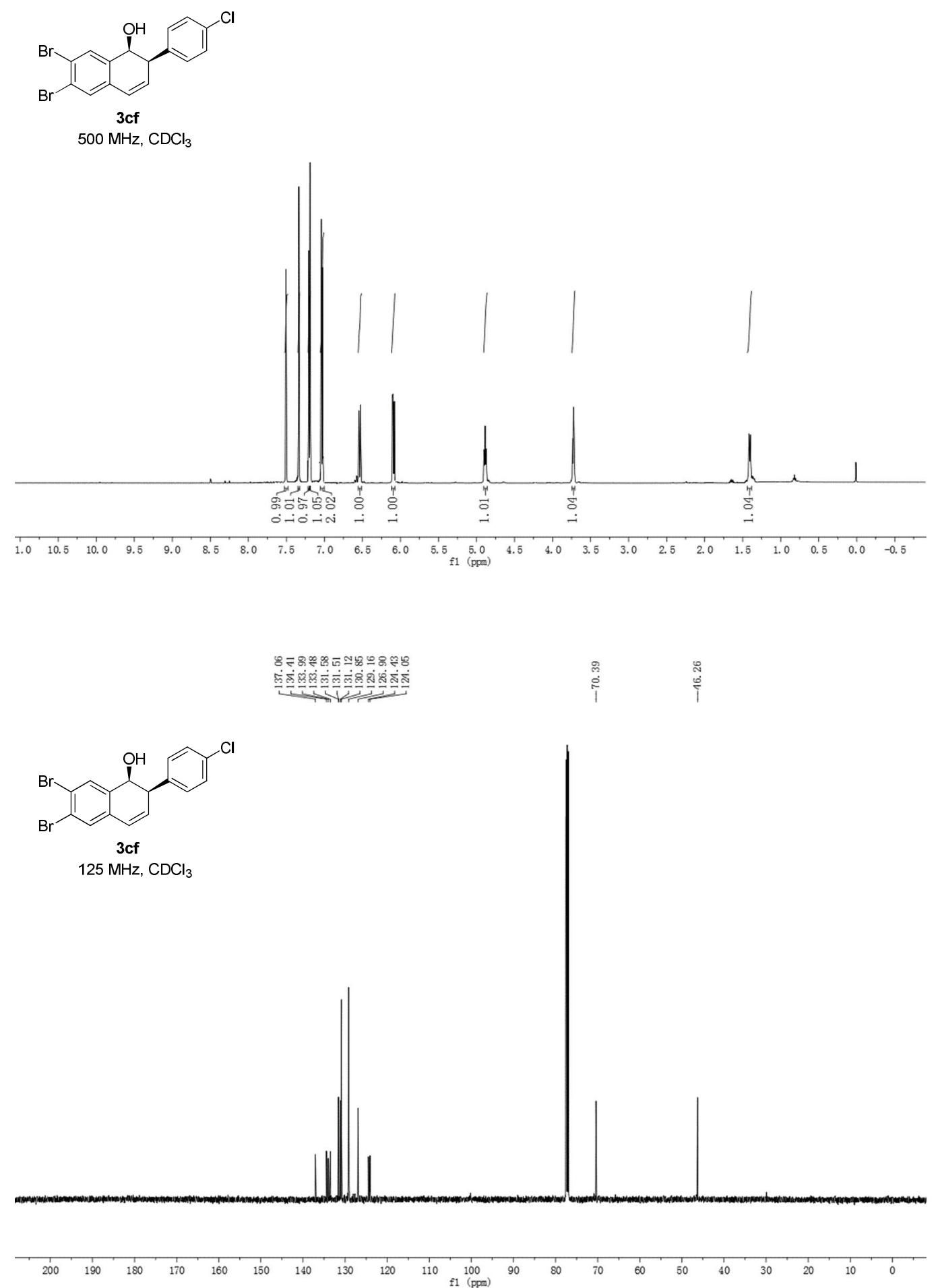


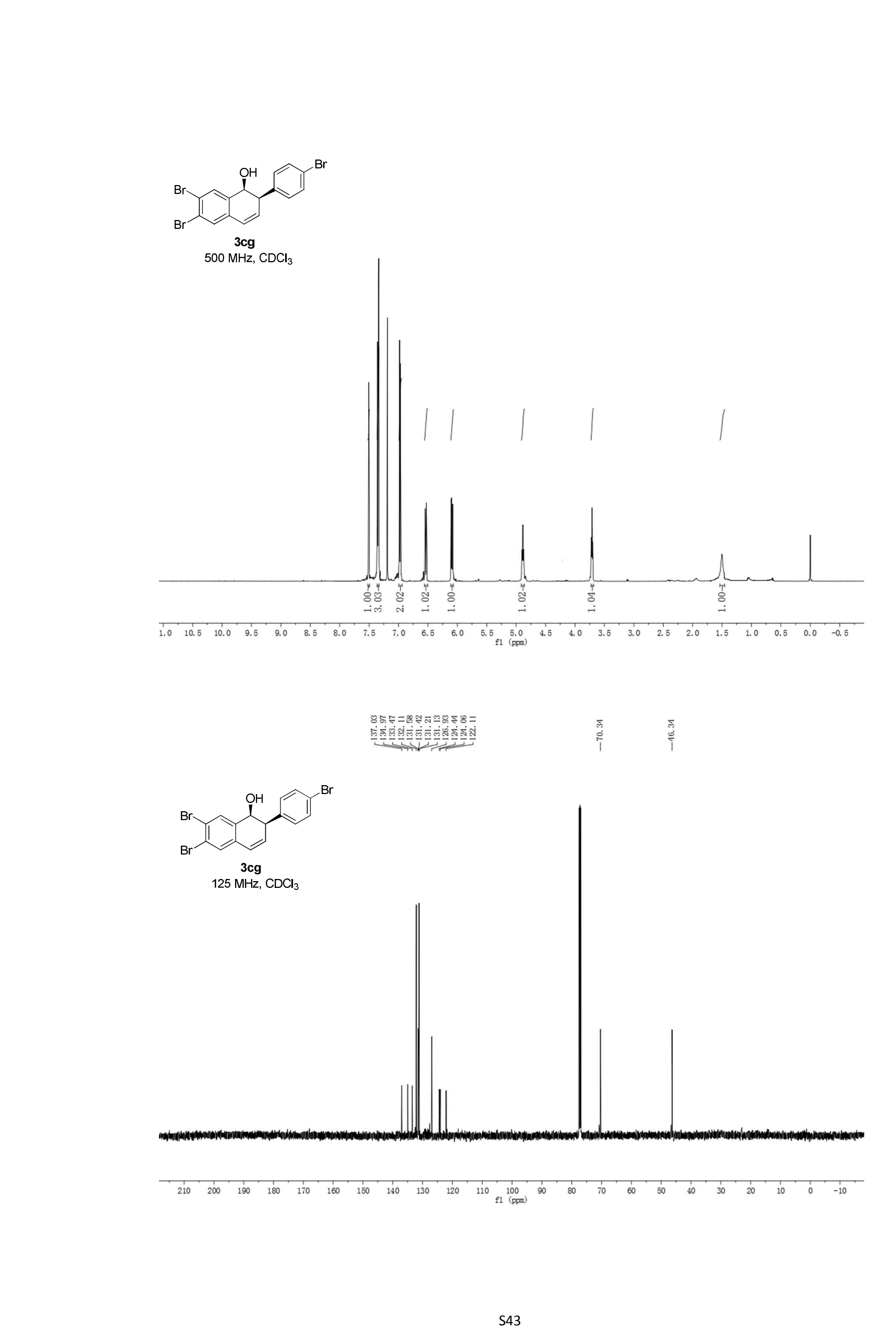



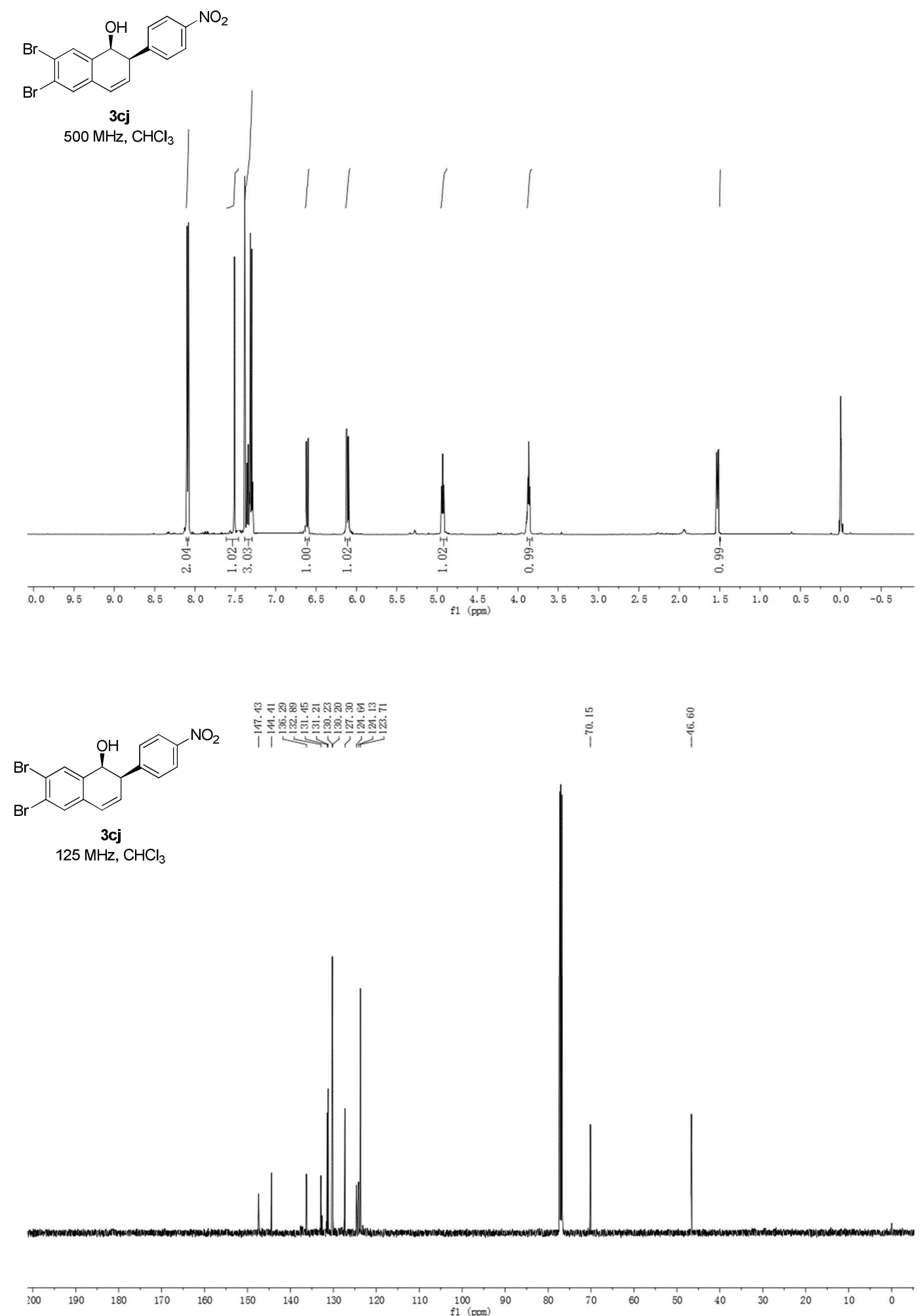

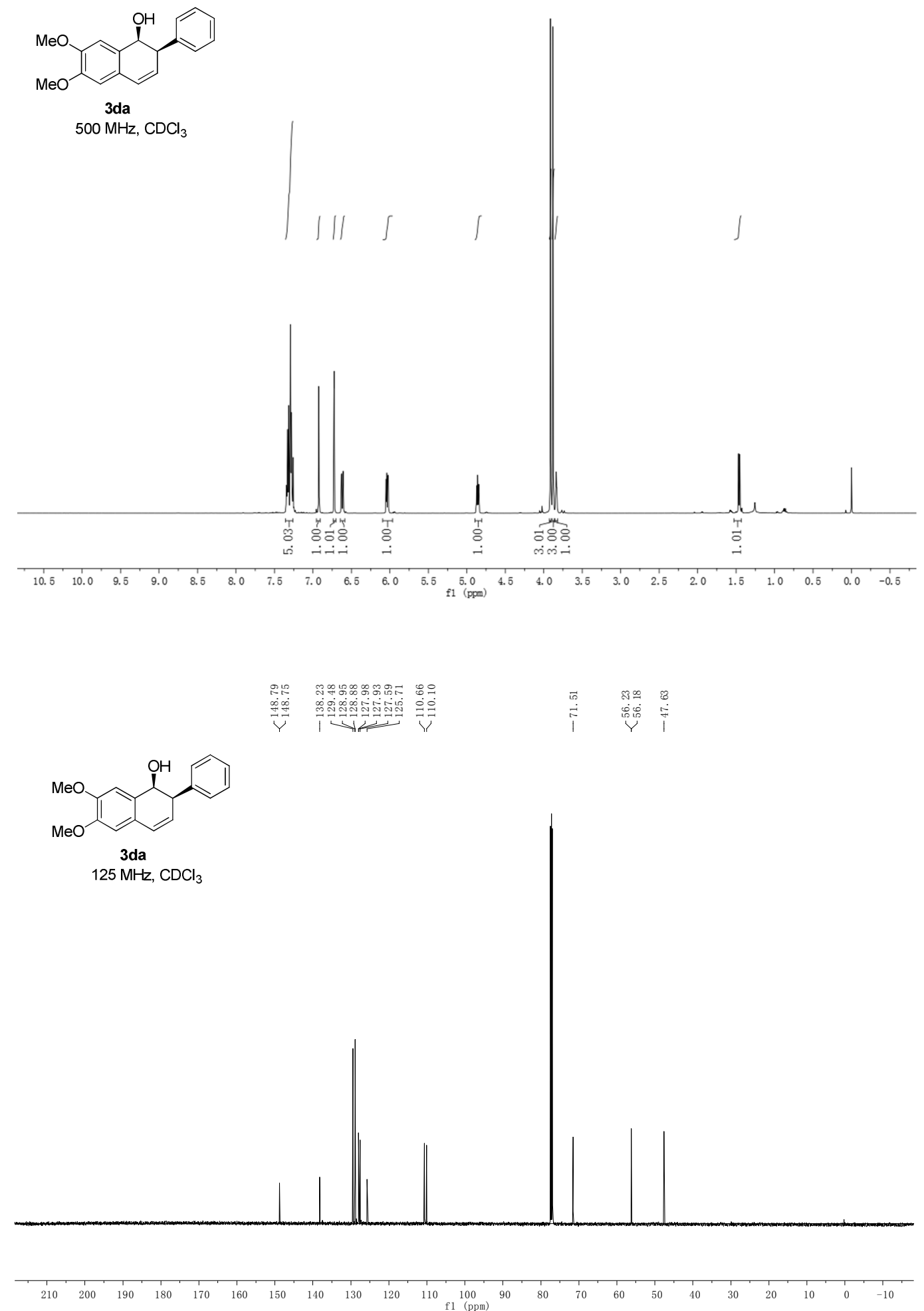

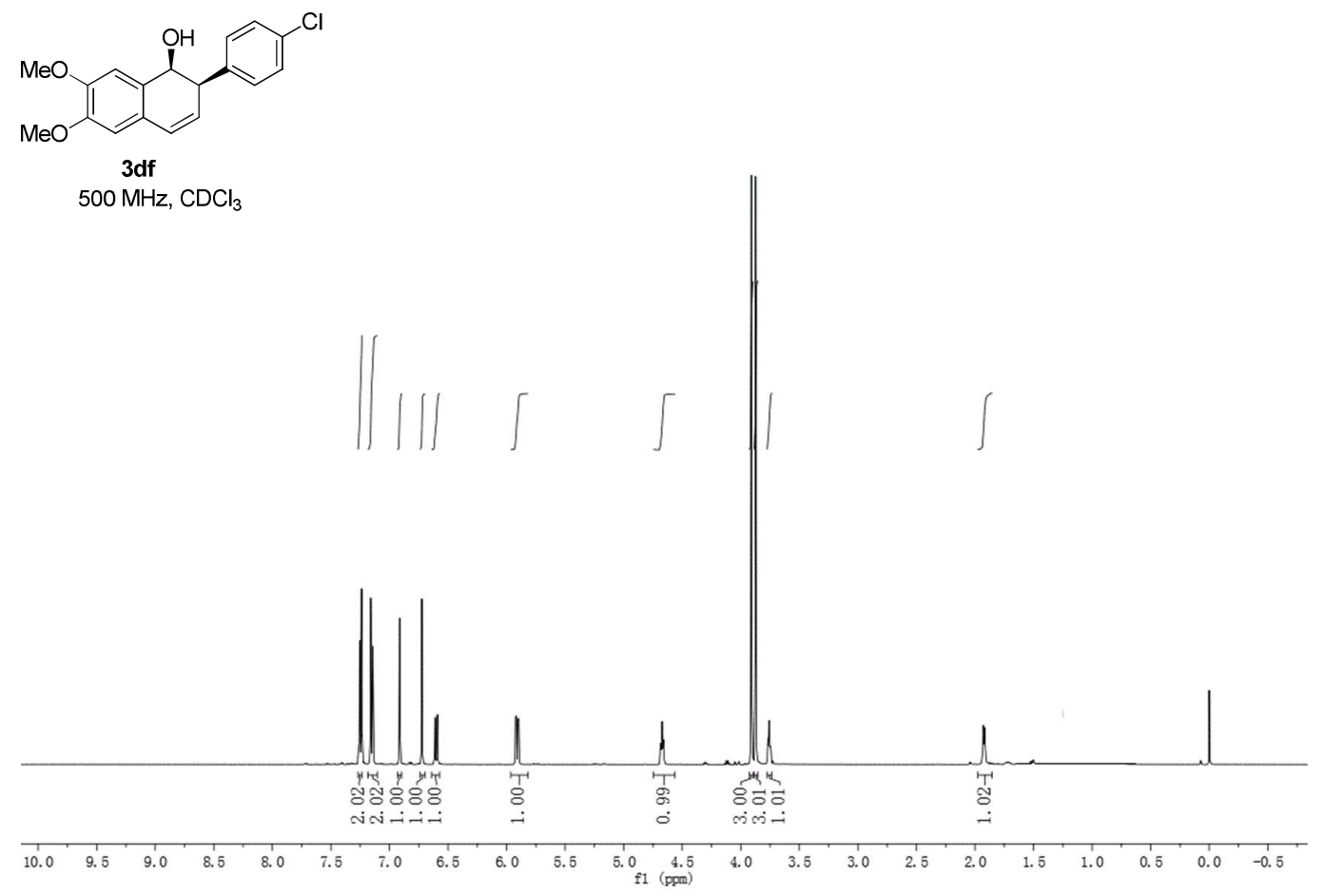

$\begin{array}{lllllllllll}10.0 & 9.5 & 9.0 & 8.5 & 8.0 & 7.5 & 7.0 & 6.5 & 6.0 & 5.5 & 5.0 \\ & & & & & & & & & & \\ \mathrm{f} 1 & (\mathrm{pg})\end{array}$

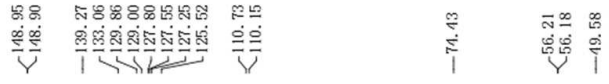
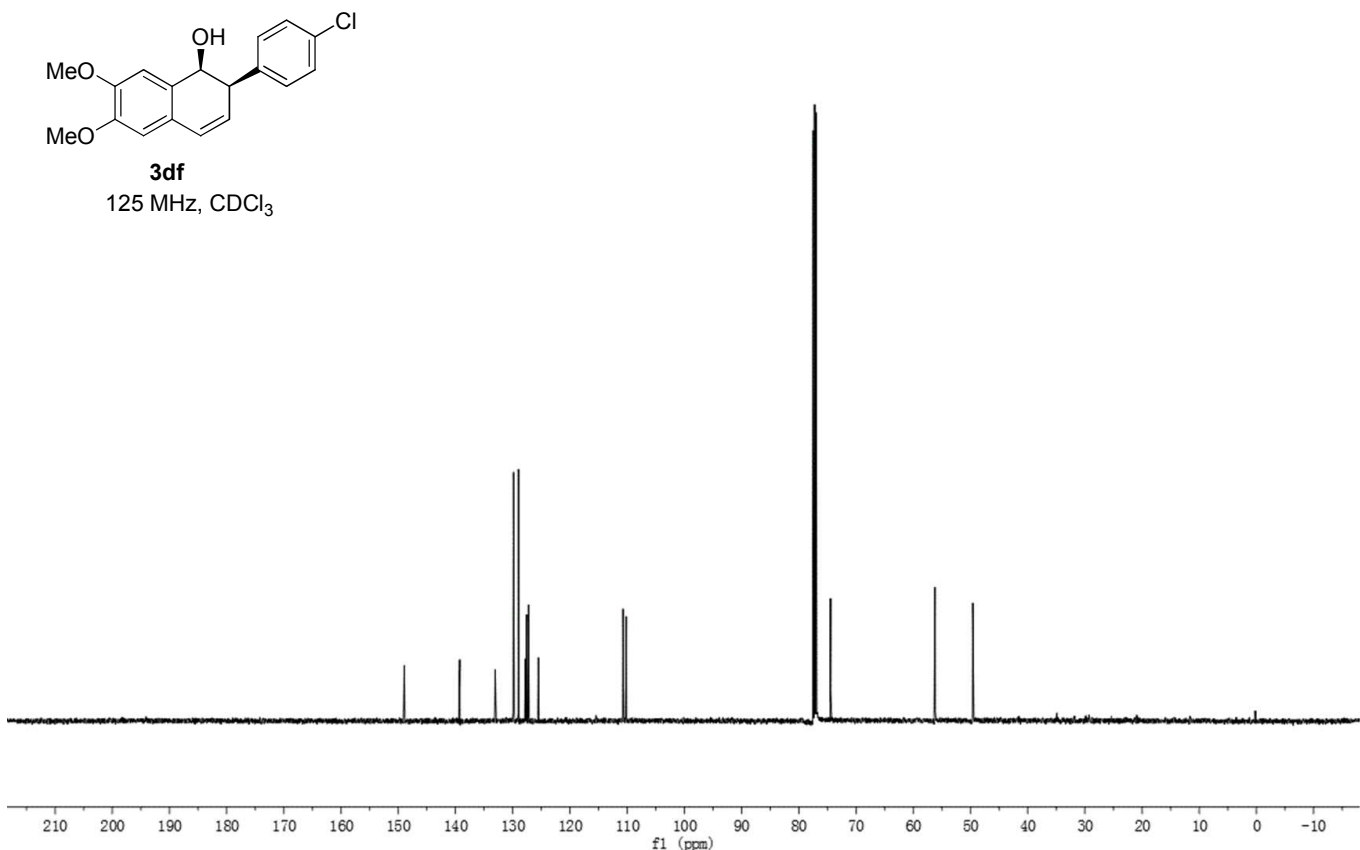

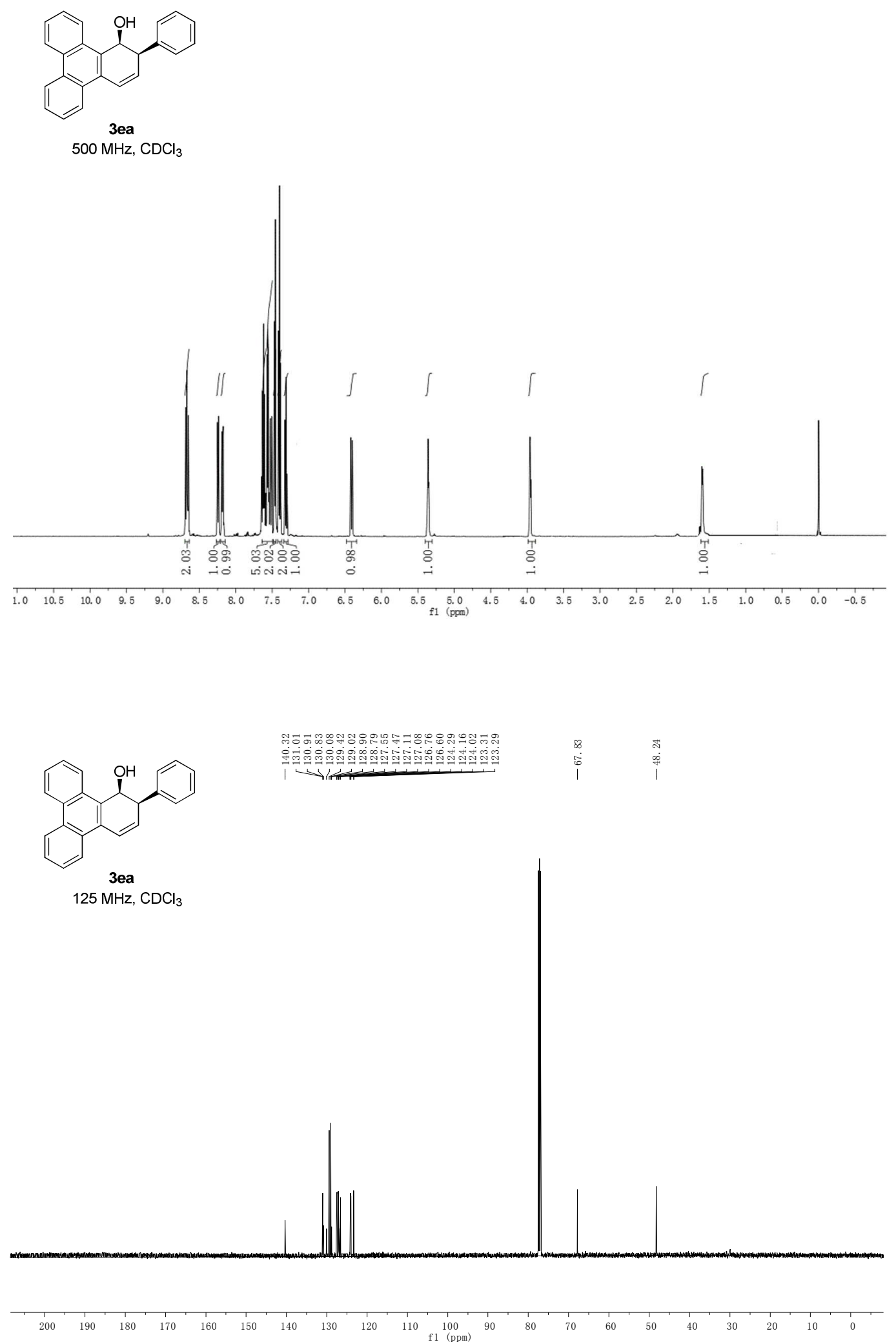